SWAT-217

UW/PT 99-04

TIFR/TH/99-02

hep-th/9902134

\title{
The BPS Spectra of Gauge Theories in Two and Four Dimensions
}

\author{
Nicholas Dorey ${ }^{1,4}$, Timothy J. Hollowood ${ }^{2,4}$ and David Tong ${ }^{3}$ \\ ${ }^{1}$ Department of Physics, University of Washington, Box 351560 \\ Seattle, Washington 98195-1560, USA \\ dorey@phys . washington . edu \\ ${ }^{2}$ Theoretical Division T-8, Los Alamos National Laboratory, \\ Los Alamos, NM 87545, USA \\ pyth@schwinger.lanl.gov \\ ${ }^{3}$ Tata Institute of Fundamental Research, \\ Homi Bhabha Rd, Mumbai, 400 005, India \\ tong@theory.tifr.res.in \\ ${ }^{4}$ Department of Physics, University of Wales, Swansea \\ Singleton Park, Swansea, SA2 8PP, UK
}

\begin{abstract}
We study $\mathcal{N}=(2,2)$ supersymmetric abelian gauge theories in two dimensions. The exact BPS spectrum of these models is shown to coincide with the spectrum of massive hypermultiplets of certain $\mathcal{N}=2$ supersymmetric gauge theories in four dimensions. A special case of these results involves a surprising connection between four-dimensional $\mathcal{N}=2$ SQCD with $N$ colours and $N_{f}>N$ flavours at the root of the baryonic Higgs branch and the supersymmetric $C P^{2 N-N_{f}-1}$ $\sigma$-model in two dimensions. This correspondence implies a new prediction for the strongcoupling spectrum of the four-dimensional theory.
\end{abstract}




\section{Introduction}

It has been noted many times in the past that two-dimensional theories with $\mathcal{N}=(2,2)$ supersymmetry have intriguing similarities to supersymmetric gauge theories in four dimensions. In this paper we will describe a quantitative correspondence between $\mathcal{N}=(2,2)$ theories in two dimensions and certain gauge theories with $\mathcal{N}=2$ supersymmetry in four dimensions which suggests new results for both types of model. In the present context, the key feature shared by these models is the existence of BPS states. The specific theories we will consider are,

Theory A: Two-dimensional $\mathcal{N}=(2,2)$ supersymmetric $U(1)_{G}$ gauge theory with dimensionful gauge coupling $e$. The matter content consists of $N$ chiral multiplets with charge +1 under $U(1)_{G}$ and twisted masses $m_{1}, \ldots, m_{N}$ [䧃]. A further $\tilde{N}<N$ chiral multiplets have charge -1 and twisted masses $\tilde{m}_{1}, \ldots, \tilde{m}_{\tilde{N}}$. The classical theory has a dimensionless FayetIliopoulis (FI) parameter $r$ and vacuum angle $\theta$ which are combined in a single complex coupling $\tau=i r+\theta / 2 \pi$. In the quantum theory, $\tau$ is eliminated in favour of a dynamical scale, $\Lambda$.

Theory B: Four-dimensional $\mathcal{N}=2 S U(N)$ SQCD with $N_{f}=N+\tilde{N}$ fundamental hypermultiplets with masses $m_{1}, \ldots, m_{N}$ and $\tilde{m}_{1}, \ldots, \tilde{m}_{\tilde{N}}$. The vacuum expectation value of the vector multiplet scalar is taken such that the theory lies at the root of its first baryonic Higgs branch. The dimensionless gauge coupling $g$ and vacuum angle $\vartheta$ combine to form the complex coupling $\tau=4 \pi i / g^{2}+\vartheta / 2 \pi$ which is a parameter of the classical theory. In the quantum theory, $\tau$ is eliminated in favour of a dynamical scale, $\Lambda$.

The main result presented below is that the BPS spectrum of Theory A is identical to the spectrum of massive BPS-saturated hypermultiplets of Theory B. By this we mean that the masses of corresponding BPS multiplets in each theory are identical as functions of the parameters $m_{i}, \tilde{m}_{\tilde{i}}$ and $\Lambda$. A simplified version of the same correspondence also holds at the classical level with dependence on $\Lambda$ replaced by dependence on $\tau$. Similar results for the special case $\tilde{N}=0$ were presented in [2]. In this paper we extend the correspondence to the general case described above and investigate the consequences for the strong-coupling spectrum of the four-dimensional theory. We also discuss the correspondence of the BPS states in terms of the intersecting brane configuration introduced in [1] and its counterpart for the four-dimensional theory 3 .

The first step in demonstrating the claimed correspondence is to show that the central

\footnotetext{
${ }^{1}$ As explained below and in [2] the BPS spectrum of Theory A does not depend on the two-dimensional gauge coupling $e$.
} 
charges of both theories are identical as functions of the parameters. The central charge of the four-dimensional model is governed by the periods of an elliptic curve which is given explicitly in [4]. In contrast, the two-dimensional central charge is determined by the critical values of a twisted superpotential. A key property of the two-dimensional BPS spectrum is its stability against D-term perturbations including, in particular, variation of the gauge coupling $e$ [5, 6]. Because of this property, the exact twisted superpotential of Theory A and therefore its exact central charge can be determined by a one-loop calculation performed at weak coupling. We find that the central charge of both models is given by the same holomorphic function of $m_{i}$, $\tilde{m}_{\tilde{i}}$ and $\Lambda$.

To check that the BPS spectra of the two theories agree, it is also necessary to specify which BPS states occur in each model. If we choose the parameters $m_{i}$ so that a sufficient number of gauge bosons have masses much greater than $|\Lambda|$, the four-dimensional theory is weakly coupled. In this case, the BPS spectrum, which can be determined by semiclassical methods, includes quarks, monopoles and dyons which are $\mathcal{N}=2$ hypermultiplets in addition to the gauge bosons which lie in $\mathcal{N}=2$ gauge multiplets. We also find a characteristic spectrum of quark-monopole boundstates which have not been described before. In fact it is easy to show that Theory A, which is also weakly-coupled in this region of parameter space, contains BPS states corresponding to each massive quark, monopole and dyon hypermultiplet of Theory B, as well as counterparts of each of the quark-monopole boundstates mentioned above.

Away from weak-coupling, the problem of determining which BPS states occur in the spectrum is complicated by the presence of curves of marginal stability on which BPS states may decay. For $\mathcal{N}=2$ theories in four dimensions, this problem has only been solved for gauge group $S U(2)$ [7]. However, in the two dimensional case, we can make some progress by noting that Theory A effectively reduces to the supersymmetric $C P^{N-\tilde{N}-1} \sigma$-model at the special point in the strong-coupling region of parameter space where all twisted masses vanish? This model is integrable and its exact spectrum is well known [8, 9, 6. The $\sigma$-model has an unbroken $S U(N-\tilde{N})$ global symmetry and the BPS states form multiplets of this symmetry. In addition to kinks transforming in the fundamental representation, there is a characteristic spectrum of boundstates transforming in antisymmetric tensor representations of $S U(N-\tilde{N})$. Small twisted masses break the global symmetry and introduce mass splittings for these multiplets which are exactly determined by the one-loop twisted superpotential described above. As the point $m_{i}=\tilde{m}_{\tilde{i}}=0$ is not singular, this description of the spectrum should be valid in some open region of the parameter space containing this point. This strong coupling spectrum of kinks and their boundstates is obviously quite different from the weak coupling spectrum

\footnotetext{
${ }^{2}$ As explained in Section 3 below, if $\tilde{N} \neq 0$ Theory A also has a massless spectrum and it is necessary to introduce certain additional complex mass parameters to reduce the theory to the $\sigma$-model in question.
} 
described above. For example the former spectrum contains only a finite number of BPS states while the latter is infinite. As usual these differences can be resolved by the presence of curves of marginal stability which disconnect the parameter space into inequivalent regions.

As the exact central charges of Theory A and Theory B are identical, the same curves of marginal stability occur in both theories. Combining this with the fact that the spectra of the two theories already agree at weak coupling, it seems very plausible that the correspondence should hold throughout the parameter space. Indeed in the $N=2$ case, where four-dimensional results are available, this agreement can be checked explicitly [2]. To strengthen this conclusion in the general case, we review the realization of Theory A [1] and Theory B [3], on the worldvolumes of intersecting M-theory branes. In either theory, BPS states appear as M2 branes whose boundaries lie on M5-branes. In both cases, the relevant boundaries naturally correspond to one-cycles on the same Riemann surface.

The equivalence of two-dimensional and four-dimensional spectra considered here has interesting consequences for both theories. As in [2], we learn that the two-dimensional theory displays many of the phenomena which are characteristic of $\mathcal{N}=2$ theories in four-dimensions. These include non-trivial monodromies of the spectrum in the complex parameter space, curves of marginal stability as well as strong-coupling vacua with massless solitons. Conversely, setting all twisted masses to zero we uncover an unexpected relation between four-dimensional $S U(N)$ $\mathcal{N}=2 \mathrm{SQCD}$ with $N_{f}=N+\tilde{N}$ massless flavours at the baryonic root and the $C P^{N-\tilde{N}-1}$ $\sigma$-model in two dimensions. Both models are asymptotically free and have a $U(1)$ R-symmetry which is broken to a $Z_{2 N-2 \tilde{N}}$ subgroup by instantons?. The global $S U(N-\tilde{N})$ symmetry of the $\sigma$-model is identified with the unbroken global symmetry on the baryonic branch of the four-dimensional theory. The correspondence described above then makes a prediction for the four-dimensional BPS spectrum at the baryonic root. In addition to the massless spectrum at the baryonic root which was described in [4], we should find massive BPS hypermultiplets transforming in the fundamental representation of the global $S U(N-\tilde{N})$ symmetry as well as boundstates in each anti-symmetric tensor representation of this group. Interestingly, an explicit semiclassical shows that these states are also present in the weak-coupling regime of the same four-dimensional theoryf.

The plan of the paper is as follows. In section 2 we discuss various classical aspects of the two dimensional theory including a description of the twisted masses, the moduli space

\footnotetext{
${ }^{3}$ Note that the correspondence discussed here is quite distinct from the more conventional analogy between the supersymmetric $C P^{N-1} \sigma$-model in two dimensions and $\mathcal{N}=1$ supersymmetric Yang-Mills theory with gauge group $S U(N)$ in four dimensions

${ }^{4}$ In other words, the theory with zero bare masses obtained from Theory B by moving away from the baryonic root to the region where the adjoint VEVs are much larger than $|\Lambda|$
} 
of classical vacua, the central charge and the spectrum of elementary quanta and solitons. In order to describe the latter in a manifestly supersymmetric manner, it is necessary to work in a dual Landau-Ginzburg formalism of the theory which we describe. In section 3, we turn to the quantum aspects of Theory $\mathrm{A}$ and determine the exact central charge and, in certain regimes of the parameter space, the BPS spectrum. In section 4, we show that the central charge of Theory A does indeed coincide with that of Theory B. We further describe the four-dimensional massive hypermultiplet spectrum in the weak-coupling regime and show that it agrees with the two-dimensional spectrum. Finally, in Section 5, we consider the IIA/M-brane description of the two theories.

\section{Theory A: Classical Analysis}

Two-dimensional gauge theories with $\mathcal{N}=(2,2)$ supersymmetry were studied in detail by Witten in [10] and an extensive introduction to these theories, including the $\mathcal{N}=(2,2)$ superspace formalism used below, can be found in this reference. Additional relevant background material is given in [1, 2]. The conventions used are those of [2]. The two-dimensional gauge theory described in the introduction is built from a vector superfield $V, N$ chiral superfields $\Phi_{i}, i=1, \ldots, N$, with charge +1 under the gauge group $U(1)_{G}$, and $\tilde{N}$ chiral superfields $\tilde{\Phi}_{\tilde{i}}$, $\tilde{i}=1, \ldots, \tilde{N}$, with charge -1 . The gauge field kinetic terms are written most simply in terms of a gauge-invariant twisted chiral superfield, $\Sigma=D_{+}^{\dagger} D_{-} V$, whose lowest component is a complex scalar $\sigma$ and also includes the $U(1)$ field strength as well as fermionic superpartners. $\Sigma$ is referred to as the field-strength superfield of the corresponding gauge multiplet $V$. Each chiral multiplet $\Phi_{i}\left(\tilde{\Phi}_{\tilde{i}}\right)$ consists of a complex scalar, $\phi_{i}\left(\tilde{\phi}_{\tilde{i}}\right)$, and a single Dirac fermion.

The kinetic terms for all fields are contained in an $\mathcal{N}=(2,2)$ D-term,

$$
\mathcal{L}_{K}=\int \mathrm{d}^{4} \theta\left[\sum_{i=1}^{N} \Phi_{i}^{\dagger} e^{2 V} \Phi_{i}+\sum_{\tilde{i}=1}^{\tilde{N}} \tilde{\Phi}_{\tilde{i}}^{\dagger} e^{-2 V} \tilde{\Phi}_{\tilde{i}}-\frac{1}{4 e^{2}} \Sigma^{\dagger} \Sigma\right]
$$

where $e$ is the dimensionful gauge coupling constant. Two dimensionless parameters will also prove important in the story: the Fayet-Iliopoulis (FI) parameter, $r$, and the vacuum angle, $\theta$. These are simply incorporated in the superfield formalism via the twisted F-term [10],

$$
\mathcal{L}_{F I}=\frac{i \tau}{2} \int \mathrm{d}^{2} \vartheta \Sigma+\text { h.c. }
$$

where $\tau=i r+\theta / 2 \pi$ and $\mathrm{d}^{2} \vartheta$ is the measure over the 'twisted' half of superspace. Both the D- and F-terms above are invariant under the full $U(1)_{A} \times U(1)_{R}$ automorphism group 
of the $\mathcal{N}=(2,2)$ supersymmetry algebra. The theory also has a global flavour symmetry $H=U(N) \times U(\tilde{N}) / U(1)_{G}$.

Gauge theories with $\mathcal{N}=(2,2)$ supersymmetry admit two different types of supersymmetric mass parameters for chiral multiplets. The first arises by dimensional reduction of the corresponding 4D mass term and has the form of an ordinary superpotential (as opposed to a twisted superpotential),

$$
\mathcal{L}_{\hat{m}}=\int d^{2} \theta \hat{m}_{i \tilde{j}} \Phi_{i} \tilde{\Phi}_{\tilde{j}}+\text { h.c. }
$$

Following [1] we refer to the parameters $\hat{m}_{i \tilde{j}}$ as complex masses. Generic non-zero complex masses break $U(1)_{R}$ and also completely break the global symmetry group $H$. The R-symmetry $U(1)_{A}$ is left unbroken. The second kind of mass parameter, known as a twisted mass, has no counterpart in four dimensions. Twisted masses are introduced by weakly gauging the global flavour symmetry $H$ of the model and constraining the lowest component of the corresponding field-strength multiplet to a fixed background expectation value. In fact, in order to preserve supersymmetry this expectation value must be diagonalisable and we will only gauge the Cartan subalgebra $C$ of the flavour group $H$. We have $C=\left(\otimes_{i=1}^{N} U(1)_{i}\right) \times\left(\otimes_{\tilde{i}=1}^{\tilde{N}} \tilde{U}(1)_{\tilde{i}}\right) / U(1)_{G}$, where $U(1)_{i}$ is defined so that the chiral multiplet $\Phi_{j}$ has charge $+\delta_{i j}$ while the $\tilde{\Phi}_{\tilde{j}}$ are neutral. Similarly, under $\tilde{U}(1)_{i}$, the $\Phi_{j}$ are neutral while the $\tilde{\Phi}_{\tilde{j}}$ carry charge $-\delta_{\tilde{i} \tilde{j}}$. Modding out by $U(1)_{G}$ is necessary because because the sum of the generators of the global $U(1)$ subgroups defined above generates the original gauge group.

The vector multiplets corresponding to the newly gauged $U(1)$ 's are denoted as $V_{i}, i=$ $1, \ldots, N$ and $\tilde{V}_{\tilde{i}}, \tilde{i}=1, \ldots, \tilde{N}$ and the D-term Lagrangian for the kinetic terms becomes

$$
\tilde{\mathcal{L}}_{K}=\int \mathrm{d}^{4} \theta\left[\sum_{i=1}^{N} \Phi_{i}^{\dagger} e^{2\left(V+V_{i}\right)} \Phi_{i}+\sum_{\tilde{i}=1}^{\tilde{N}} \tilde{\Phi}_{\tilde{i}}^{\dagger} e^{-2\left(V+\tilde{V}_{\tilde{i}}\right)} \tilde{\Phi}_{\tilde{i}}-\frac{1}{4 e^{2}} \Sigma^{\dagger} \Sigma\right]
$$

As each of their component fields is to be constrained, a kinetic term for the new gauge fields would be redundant. Instead we introduce a twisted chiral multiplet, $\Lambda_{i}\left(\tilde{\Lambda}_{\tilde{i}}\right)$, for each new gauge multiplet, $V_{i}\left(\tilde{V}_{\tilde{i}}\right)$, adding to the Lagrangian the term,

$$
\mathcal{L}_{L M}=\frac{i}{2} \int \mathrm{d}^{2} \vartheta\left[\sum_{i=1}^{N} \Lambda_{i}\left(\Sigma_{i}-m_{i}\right)+\sum_{\tilde{i}=1}^{\tilde{N}} \tilde{\Lambda}_{\tilde{i}}\left(\tilde{\Sigma}_{\tilde{i}}-\tilde{m}_{\tilde{i}}\right)\right]+\quad \text { h.c. }
$$

where $\Sigma_{i}\left(\tilde{\Sigma}_{\tilde{i}}\right)$ is the field strength superfield for $V_{i}\left(\tilde{V}_{\tilde{i}}\right)$. These twisted chiral superfields act as Lagrange multipliers for the constraints $\Sigma_{i}=m_{i}$ and $\tilde{\Sigma}_{\tilde{i}}=\tilde{m}_{\tilde{i}}$. They also constrain the corresponding gauge fields to be pure gauge. Since these field strengths are themselves twisted 
chiral superfields, $m_{i}$ and $\tilde{m}_{\tilde{i}}$ are referred to as twisted masses. In addition to breaking $H$ down to its Cartan subalgebra, generic non-zero twisted masses also break $U(1)_{A}$. The residual R-symmetry group in the presence of twisted masses is $U(1)_{R}$. Note that the above procedure for introducing twisted masses only makes sense if the subgroup of the global symmetry group which we are gauging is unbroken. For this reason, if the chiral field $\Phi_{i}$ appears in a complex mass term such as (2.2), it cannot also have a non-zero twisted mass?. In this paper we will be primarily interested in the theory with non-zero twisted masses and complex masses are henceforth set to zero unless stated otherwise. The superspace Lagrangian for the model of interest is therefore $\mathcal{L}=\tilde{\mathcal{L}}_{K}+\mathcal{L}_{F I}+\mathcal{L}_{L M}$. Note also that the sum of all twisted masses may be changed by a shift in $\sigma$ and we use this freedom to set $\sum_{i=1}^{N} m_{i}=0$ while leaving the masses $\tilde{m}_{\tilde{i}}$ unconstrained.

An alternative expression for this Lagrangian which will prove useful below is $\mathcal{L}=\mathcal{L}_{D}+\mathcal{L}_{F}$ where the D-term is written as,

$\mathcal{L}_{D}=\int d^{4} \theta \sum_{i=1}^{N}\left[\exp \left(X_{i}+X_{i}^{\dagger}+2 U_{i}\right)-2 \mathcal{R}_{i} U_{i}\right]+\sum_{\tilde{i}=1}^{\tilde{N}}\left[\exp \left(\tilde{X}_{\tilde{i}}+\tilde{X}_{\tilde{i}}^{\dagger}-2 \tilde{U}_{\tilde{i}}\right)-2 \tilde{\mathcal{R}}_{i} \tilde{U}_{\tilde{i}}\right]-\frac{1}{4 e^{2}} \Sigma^{\dagger} \Sigma$

with $\Phi_{i}=\exp \left(X_{i}\right)$ and $\tilde{\Phi}_{\tilde{i}}=\exp \left(\tilde{X}_{\tilde{i}}\right)$. In the above expression we have also redefined the gauge superfields as $U_{i}=V+V_{i}$ and $\tilde{U}_{\tilde{i}}=V+\tilde{V}_{\tilde{i}}$ and split the Lagrange multiplier superfields into real and imaginary parts according to,

$$
\Lambda_{i}=i \mathcal{R}_{i}+\frac{\Theta_{i}}{2 \pi} \quad \tilde{\Lambda}_{i}=i \tilde{\mathcal{R}}_{\tilde{i}}+\frac{\tilde{\Theta}_{\tilde{i}}}{2 \pi}
$$

Notice that each gauge field $U_{i}\left(\tilde{U}_{\tilde{i}}\right)$ has Fayet-Iliopoulis term where the FI coupling is the imaginary part, $\mathcal{R}_{i}\left(\tilde{\mathcal{R}}_{\tilde{i}}\right)$, of the twisted chiral superfield $\Lambda_{i}\left(\tilde{\Lambda}_{\tilde{i}}\right)$. Note also that we have chosen to write these terms in the conventional way as D-terms depending on the vector superfields $U_{i}$ $\left(\tilde{U}_{\tilde{i}}\right)$ rather than as twisted F-terms analogous to (2.1) above which involve the corresponding field-strength superfields. The residual twisted F-terms are conveniently written in terms of a twisted superpotential as

$$
\mathcal{L}_{F}=\int d^{2} \vartheta \mathcal{W}\left(\Lambda_{i}, \tilde{\Lambda}_{\tilde{i}}, \Sigma\right)+\int d^{2} \bar{\vartheta} \overline{\mathcal{W}}\left(\Lambda_{i}^{\dagger}, \tilde{\Lambda}_{\tilde{i}}^{\dagger}, \Sigma^{\dagger}\right)
$$

with,

$$
\mathcal{W}=\frac{i}{2}\left[\tau \Sigma-\sum_{i=1}^{N} \Lambda_{i}\left(m_{i}+\Sigma\right)-\sum_{\tilde{i}=1}^{\tilde{N}} \tilde{\Lambda}_{\tilde{i}}\left(\tilde{m}_{\tilde{i}}+\Sigma\right)\right]
$$

\footnotetext{
${ }^{5}$ More precisely the twisted masses of the two chiral superfields appearing in (2.2) are not independent parameters but obey a certain linear constraint.
} 
At this point we have a model which contains both chiral and twisted chiral superfields. These two kinds of superfield have exactly the same content in terms of component fields and it is sometimes possible to eliminate a chiral superfield in favour of a twisted chiral superfield (or vice versa) using the two-dimensional duality transformation introduced by Rocek and Verlinde [11]. In the following we will use this transformation to derive a dual formulation in which the Lagrangian has a simple Landau-Ginzburg form. This is particularly straightforward for the present model as the first step in the duality transformation of [11] is to gauge a single global symmetry generator for each chiral superfield exactly as we have done above. Further in the usual duality transformation one must also introduce a Lagrange multiplier to enforce the constraint that the new gauge fields are pure gauge. This step also has already been performed above in the process of introducing twisted masses. The fact that the scalar components of these gauge multiplets are constrained to a non-zero value in the present case means this is a slight generalization of the usual duality transformation.

To complete the duality transformation requires two more steps. First the chiral multiplets $X_{i}$ and $\tilde{X}_{\tilde{i}}$ are absorbed by the gauge transformation,

$$
\begin{aligned}
& U_{i} \rightarrow U_{i}-\frac{1}{2}\left(X_{i}+X_{i}^{\dagger}\right) \\
& \tilde{U}_{\tilde{i}} \rightarrow \tilde{U}_{\tilde{i}}+\frac{1}{2}\left(\tilde{X}_{\tilde{i}}+\tilde{X}_{\tilde{i}}^{\dagger}\right)
\end{aligned}
$$

Second we can eliminate the gauge multiplets $U_{i}$ and $\tilde{U}_{\tilde{i}}$ via their equations of motion which read,

$$
\exp \left(2 U_{i}\right)=\mathcal{R}_{i} \quad \exp \left(-2 \tilde{U}_{\tilde{i}}\right)=-\tilde{\mathcal{R}}_{\tilde{i}}
$$

The result is a dual description of the theory in terms of the chiral superfields $\Lambda_{i}, \tilde{\Lambda}_{\tilde{i}}$ and $\Sigma$ with Lagrangian of Landau-Ginzburg form. We have $\mathcal{L}=\mathcal{L}_{D}+\mathcal{L}_{F}$ with,

$$
\mathcal{L}_{D}=\int d^{4} \theta \mathcal{K}\left[\Lambda_{i}, \Lambda_{i}^{\dagger}, \tilde{\Lambda}_{\tilde{i}}, \tilde{\Lambda}_{\tilde{i}}^{\dagger}, \Sigma, \Sigma^{\dagger}\right]
$$

where the Kähler potential is given by,

$$
\mathcal{K}=\sum_{i=1}^{N} \mathcal{R}_{i}\left(1-\log \mathcal{R}_{i}\right)-\sum_{\tilde{i}=1}^{\tilde{N}} \tilde{\mathcal{R}}_{\tilde{i}}\left(1-\log \left(-\tilde{\mathcal{R}}_{i}\right)\right)-\frac{1}{4 e^{2}} \Sigma^{\dagger} \Sigma
$$

The twisted F-term Lagrangian $\mathcal{L}_{F}$ is defined in (2.7,2.8) above. This dual formulation of the model will be particularly useful when discussing the properties of BPS solitons below. A limit of the theory which will be of particular interest below is the strong-coupling limit $e \rightarrow \infty$. In this limit the kinetic terms for the gauge multiplet can be omitted from the Kähler potential 
(2.12) and the field-strength multiplet $\Sigma$ can be eliminated via its equation of motion which imposes the linear constraint,

$$
\sum_{i=1}^{N} \Lambda_{i}+\sum_{\tilde{i}}^{\tilde{N}} \tilde{\Lambda}_{\tilde{i}}=\tau
$$

Returning to the original formulation of the model, with Lagrangian $\mathcal{L}=\tilde{\mathcal{L}}_{K}+\mathcal{L}_{F I}+\mathcal{L}_{L M}$ defined above, we obtain the classical scalar potential by integrating out auxiliary fields to get,

$$
U=e^{2}\left(\sum_{i=1}^{N}\left|\phi_{i}\right|^{2}-\sum_{\tilde{i}=1}^{\tilde{N}}\left|\tilde{\phi}_{\tilde{i}}\right|^{2}-r\right)^{2}+\sum_{i=1}^{N}\left|\sigma+m_{i}\right|^{2}\left|\phi_{i}\right|^{2}+\sum_{\tilde{i}=1}^{\tilde{N}}\left|\sigma+\tilde{m}_{\tilde{i}}\right|^{2}\left|\tilde{\phi}_{\tilde{i}}\right|^{2}
$$

The manifold of classical supersymmetric vacua, determined by the condition $U=0$, depends on the parameters $r, m_{i}$ and $\tilde{m}_{\tilde{i}}$. We begin by considering the case of zero twisted masses, $m_{i}=\tilde{m}_{\tilde{i}}=0$. In this case the theory has a classical Higgs branch with $\sigma=0$, determined by solving the equation,

$$
\sum_{i=1}^{N}\left|\phi_{i}\right|^{2}-\sum_{\tilde{i}=1}^{\tilde{N}}\left|\tilde{\phi}_{\tilde{i}}\right|^{2}=r
$$

modulo $U(1)$ gauge transformations. The quotient of the solution space of (2.15) by $U(1)_{G}$ defines a toric variety, $\mathcal{M}_{H}(r)$, of complex dimension $N+\tilde{N}-1$ [10, 12]. The generators of the global symmetry group $\mathrm{H}$ act as isometries on $\mathcal{M}_{H}$. For $r>0(r<0) \mathcal{M}_{H}$ has Kähler class $r(-r)$ and first Chern class $c_{1}=N-\tilde{N}(\tilde{N}-N)$. The two regions $r<0$ and $r>0$ are separated by the point $r=0$ at which the classical Higgs branch is singular. A special case is $N=\tilde{N}$, where the two regions yield a pair of birationally equivalent Calabi-Yau manifolds [10]. The classical Higgs branch is always non-compact except in the two cases, $\tilde{N}=0$ with $r>0$, and, $N=0$ with $r<0$, where the toric varieties in question are the complex projective spaces $C P^{N-1}$ and $C P^{\tilde{N}-1}$. Another special feature of the cases with $N=0(\tilde{N}=0)$, is that the classical theory has no supersymmetric vacua at all if $r$ is negative (positive). As we will see below, this and several other features of the classical theories are modified by quantum corrections. For $r=0$ only, the classical theory also has a Coulomb branch on which $\phi_{i}=\tilde{\phi}_{\tilde{i}}=0$ and $\sigma$ is unconstrained.

When generic non-zero twisted masses are introduced the classical Higgs branch is lifted leaving only a finite number of isolated supersymmetric vacua. In particular, for $r>0$, we find $N$ such vacua, $\mathcal{V}_{i}$ with $i=1,2, \ldots, N$. In the vacuum $\mathcal{V}_{j}$ the scalar fields take values $\sigma=-m_{j}, \phi_{i}=\sqrt{r} \delta_{i j}$ and $\tilde{\phi}_{\tilde{i}}=0$. Correspondingly, for $r<0$ we find $\tilde{N}$ supersymmetric vacua, 
$\tilde{\mathcal{V}}_{\tilde{i}}$ with $\tilde{i}=1,2, \ldots, \tilde{N}$. In the vacuum $\tilde{\mathcal{V}}_{\tilde{j}}$ the scalar fields take values $\sigma=-\tilde{m}_{j}, \phi_{i}=0$, and $\tilde{\phi}_{\tilde{i}}=\sqrt{-r} \delta_{\tilde{i} \tilde{j}}$. In each of these vacua the scalar component of a single chiral multiplet is non-zero and the phase of this field has been set to zero by a $U(1)_{G}$ gauge rotation. When two or more twisted masses coincide a continuous vacuum degeneracy is restored. If $r>0$, the two minimal cases occur when $m_{i}=m_{j}$ for some $i$ and $j$ and when $m_{i}=\tilde{m}_{\tilde{j}}$ for some $i$ and $\tilde{j}$. Both cases give rise to Higgs branches of complex dimension one. In the first case the Higgs branch is a copy of $C P^{1}$ while in the second case it is a non-compact complex manifold which satisfies the Calabi-Yau condition $c_{1}=0$. In contrast, the condition $\tilde{m}_{\tilde{i}}=\tilde{m}_{\tilde{j}}$ for some $\tilde{i}$ and $\tilde{j}$ does not increase the vacuum degeneracy (for $r>0$ ). As in the massless case, the theory with $r=0$ also has a Coulomb branch on which all the chiral multiplet scalars vanish and $\sigma$ is unconstrained.

We will now consider the BPS spectrum of the classical theory introduced above beginning with the case of vanishing twisted masses. In this case the full R-symmetry group $U(1)_{A} \times U(1)_{R}$ is unbroken. This implies that the $\mathcal{N}=(2,2)$ SUSY algebra has vanishing central charge and thus there are no massive BPS states in the classical theory. However, the classical theory does have massless particles corresponding to the flat directions of the Higgs and Coulomb branches described above. In particular there are $N+\tilde{N}-1$ massless chiral multiplets which correspond to complex coordinates on the classical Higgs branch $\mathcal{M}_{H}(r)$. In any open region of $\mathcal{M}_{H}$ in which $\Phi_{j} \neq 0$, the gauge-invariant superfields $W_{i}^{(j)}=\Phi_{i} / \Phi_{j}$, for $i=1,2, \ldots, N$ with $i \neq j$, together with $\tilde{W}_{\tilde{i}}^{(j)}=\Phi_{j} \tilde{\Phi}_{\tilde{i}}$, for $\tilde{i}=1,2, \ldots, N$, provide a convenient basis for these massless fields. In the classical theory the scalar components of some of these multiplets are Goldstone bosons for the broken generators of the global symmetry group $\mathrm{H}$, a situation which cannot persist in the corresponding two-dimensional quantum theory [13]. In contrast, fluctuations of the fields which are orthogonal to the vacuum manifold get masses of order $\sqrt{e}|r|$ from the Higgs mechanism. Provided $r \neq 0$, the massive fields decouple in the infra-red (IR) limit $e \rightarrow \infty$ and the resulting theory is precisely a supersymmetric non-linear $\sigma$-model with target manifold $\mathcal{M}_{H}(r)$. The coupling constant of the low-energy $\sigma$-model is related to the FI parameter as $g=\sqrt{2 / r}$.

In the presence of twisted masses, the same IR limit yields a massive deformation of the $\sigma$-model which was studied in detail for the case $\tilde{N}=0$ where $\mathcal{M}_{H}=C P^{N-1}$ in [2]. In the deformed model, the classical vacuum degeneracy is lifted as described above and each of the $N+\tilde{N}-1$ massless chiral multiplets of the $\sigma$-model acquires a mass. As usual, the conversion of massless degrees of freedom to massive is only consistent if the latter states are BPS saturated and therefore lie in short multiplets of supersymmetry. This is possible due to the fact that twisted masses break $U(1)_{A}$ allowing a non-zero central charge in the supersymmetry algebra. Further the twisted masses also break $H$ to its maximal torus and the unbroken Cartan generators are natural candidates for the central charges in question [1]. 
Massive particles which carry the global $U(1)$ charges can then form short multiplets. In the vacuum $\mathcal{V}_{j}$, the $N+\tilde{N}-1$ massless chiral multiplets $W_{i}^{(j)}$ and $\tilde{W}_{\tilde{i}}^{(j)}$ defined above get masses $\left|m_{i}-m_{j}\right|$ and $\left|\tilde{m}_{\tilde{i}}-m_{j}\right|$ respectively. Summing over each of the vacua $\mathcal{V}_{i}$ of the $r>0$ theory, we find a total of $N(N+\tilde{N}-1)$ states with distinct masses. Introducing a little new notation allows these masses to be written in a simple universal form. We denote the charge carried by a field under the global $U(1)_{k}\left(\tilde{U}(1)_{\tilde{k}}\right)$ flavour symmetries as $S_{k}\left(\tilde{S}_{\tilde{k}}\right)$ Note that $W_{i}^{(j)}=\Phi_{i} / \Phi_{j}$ then carries charges $S_{k}=\delta_{i k}-\delta_{j k}$ and $\tilde{S}_{\tilde{k}}=0$, while the field $\tilde{W}_{\tilde{i}}^{(j)}=\tilde{\Phi}_{\tilde{i}} \Phi_{j}$ carries charges $S_{k}=\delta_{j k}$ and $\tilde{S}_{\tilde{k}}=-\delta_{\tilde{i} \tilde{k}}$. The masses of all these states obey the BPS mass formula $M=\left|Z_{S}\right|$ where the corresponding central charge is,

$$
Z_{S}=i \sum_{i=1}^{N} m_{i} S_{i}+i \sum_{\tilde{i}=1}^{\tilde{N}} \tilde{m}_{\tilde{i}} \tilde{S}_{\tilde{i}}
$$

The BPS states described above are elementary quanta of the fields appearing in the Lagrangian of the mass-deformed $\sigma$-model. As the classical theory with non-zero twisted masses has isolated supersymmetric vacua, an additional possibility arises: there can be BPS saturated kinks which interpolate between distinct supersymmetric vacua at left and right spatial infinity. For $r>0$, we can define topological charges $T_{i}, i=1, \ldots, N$, such that a field configuration that tends asymptotically to the vacuum $\mathcal{V}_{j}$ as $x \rightarrow \infty$ and to $\mathcal{V}_{k}$ as $x \rightarrow-\infty$ has topological charge $T_{i}=\delta_{i j}-\delta_{i k}$. Correspondingly, for $r<0$, we define topological charges, $\tilde{T}_{\tilde{i}}$, for $\tilde{i}=1,2, \ldots, \tilde{N}$, associated with solitons which interpolate between the vacua $\tilde{\mathcal{V}}_{\tilde{i}}$. Like the global generators $S_{i}$ and $\tilde{S}_{\tilde{i}}$ defined above, the topological charges $T_{i}$ and $\tilde{T}_{\tilde{i}}$, can also contribute to the central charge [14]. Solitons with non-zero topological charges can then give rise to additional BPS states in the spectrum. As we review below, the topological contribution to the classical central charge (2.16) is included by the replacement $S_{i} \rightarrow S_{i}+\tau T_{i}$ in (2.16) for $r>0$ and $\tilde{S}_{\tilde{i}} \rightarrow \tilde{S}_{\tilde{i}}+\tau \tilde{T}_{\tilde{i}}$ for $r<0$.

For the case of the mass-deformed $C P^{N-1} \sigma$-model, BPS saturated kinks were studied in detail in [2]. We will begin by briefly reviewing the simplest example, $N=2, \tilde{N}=0$, where the target space is $C P^{1}$. In this case there are two classical vacua $\mathcal{V}_{1}$ and $\mathcal{V}_{2}$, with a single chiral multiplet of mass $|m|=\left|m_{1}-m_{2}\right|$ in each. The scalar components of these multiplets are $w=\phi_{1} / \phi_{2}$ in $\mathcal{V}_{1}$ and $1 / w$ in $\mathcal{V}_{2}$. The theory has a single global $U(1)$ charge $S=\left(S_{1}-S_{2}\right) / 2$ and a single topological charge $T=\left(T_{1}-T_{2}\right) / 2$. It will be useful to exhibit the soliton solutions in both versions of the model introduced above. Starting from the original formulation of the theory, with Lagrangian $\mathcal{L}=\tilde{\mathcal{L}}_{K}+\mathcal{L}_{F I}+\mathcal{L}_{L M}$ defined in (2.1,2.3,2.4), we take the IR limit $e \rightarrow \infty$ and eliminate the gauge field multiplet by its equations of motion. We then obtain a Lagrangian for the complex scalar $w=\phi_{1} / \phi_{2}$ and its superpartner. It is convenient to 
decompose the complex field $w$ in terms of its modulus and argument as,

$$
w=\tan \frac{\varphi}{2} \exp (i \alpha)
$$

where, in order to make the mapping one-to-one, we make the identifications $\varphi \sim \varphi+2 \pi$ and $\alpha \sim \alpha+2 \pi$. In terms of the new variables, the bosonic terms in the Lagrangian read [2],

$$
\mathcal{L}_{\text {Bose }}=-\frac{r}{4}\left[\left(\partial_{\mu} \varphi\right)^{2}+\sin ^{2} \varphi\left(|m|^{2}-\left(\partial_{\mu} \alpha\right)^{2}\right)\right]+\frac{\theta}{4 \pi} \epsilon^{\mu \nu} \partial_{\mu}(\cos \varphi) \partial_{\nu} \alpha
$$

This is a variant of the sine-Gordon (SG) Lagrangian with an additional massless field $\alpha$ which has derivative couplings to the SG field $\varphi$. The two SUSY vacua found above correspond to the two sets of zeros of the SG potential, which occur at $\varphi=2 n \pi$ and at $\varphi=(2 n+1) \pi$ for integer $n$. As $\alpha$ appears only through its derivatives it can take any constant value in the vacuum.

The classical equations of motion coming from (2.18), have a family of solutions of topological charge $T=1$, with $\alpha=\omega t$ and,

$$
\varphi=2 \tan ^{-1}\left(\exp \sqrt{|m|^{2}-\omega^{2}}\right)
$$

which are labelled by the real parameter $\omega$ with $|\omega|<|m|$. The mass and global charge of the solution are,

$$
M=\frac{r|m|^{2}}{\sqrt{|m|^{2}-\omega^{2}}} \quad S=\frac{r \omega}{\sqrt{|m|^{2}-\omega^{2}}}-\frac{\theta}{2 \pi}
$$

Applying semiclassical Bohr-Sommerfeld quantization to these periodic classical solutions we find that the allowed values of $\omega$ are quantized so that $S$ takes only integer values [2]. Hence the theory has an infinite tower of 'dyons' which carry both topological charges and Noether charges. The contribution of the vacuum angle $\theta$ to the global charge is a two-dimensional analog of the Witten effect 15 for dyons in four dimensions. Eliminating the variable $\omega$ we find that the soliton mass can be written as

$$
M=|m| \sqrt{\left(S+\frac{\theta}{2 \pi}\right)^{2}+r^{2}}
$$

¿From the above we learn that the masses of both the dyons and the elementary quanta are consistent with the BPS mass formula $M=|Z|$ where,

$$
Z=i m(S+\tau T)
$$

The description of the solitons given above and in [2] has a serious drawback: supersymmetry is not manifest and properties such as BPS saturation of the solutions have to be checked by 
explicit calculation. In particular, we would like to derive the formula (2.22) for the central charge directly, rather than guessing it from the mass spectrum as we did above. In fact for a large class of $\mathcal{N}=(2,2)$ models with Lagrangians of Landau-Ginzburg form, the Bogomol'nyi bound can be derived directly in superspace [16]. As we showed above, the two-dimensional theory with twisted masses can be be put in this standard form by performing a Rocek-Verlinde duality transformation. In the case $N=2, \tilde{N}=0$, after imposing the constraint (2.13), the dual Lagrangian can be written in terms of a single twisted chiral superfield, by setting $\Lambda_{1}=\Lambda$ and $\Lambda_{2}=\tau-\Lambda$. The resulting Lagrangian is

$$
\mathcal{L}=\int d^{4} \theta \mathcal{K}\left[\Lambda, \Lambda^{\dagger}\right]+\int d^{2} \vartheta \mathcal{W}(\Lambda)+\int d^{2} \bar{\vartheta} \overline{\mathcal{W}}\left(\Lambda^{\dagger}\right)
$$

with Kähler potential.

$$
\mathcal{K}[\Lambda]=\mathcal{R}(1-\log \mathcal{R})+(r-\mathcal{R})(1-\log (r-\mathcal{R}))
$$

where $\Lambda=i \mathcal{R}+\Theta / 2 \pi$. In the following, $\Lambda, \mathcal{R}$ and $\Theta$ will denote either the superfield or its scalar component depending on context. The Kähler metric is,

$$
g_{\Lambda \bar{\Lambda}}=-\frac{\partial^{2} \mathcal{K}}{\partial \Lambda \partial \Lambda^{\dagger}}=\frac{r}{4 \mathcal{R}(r-\mathcal{R})}
$$

and the twisted superpotential $\mathcal{W}$ has the simple form $\mathcal{W}=i m \Lambda / 2$. The Lagrangian (2.23) is in fact the most general Lagrangian for a single twisted chiral superfield with at most two derivatives and we will meet it again with different choices for $\mathcal{K}$ and $\mathcal{W}$ in the next section. For this reason we will now give a general derivation of the Bogomol'nyi bound at the same time as giving the specific formulae which relate to the present choice of $\mathcal{K}$ and $\mathcal{W}$.

After integrating out auxiliary fields the bosonic part of the above Lagrangian becomes,

$$
\begin{aligned}
\mathcal{L}_{\text {Bose }} & =-g_{\Lambda \bar{\Lambda}} \partial_{\mu} \Lambda \partial^{\mu} \Lambda^{\dagger}-g^{\Lambda \bar{\Lambda}} \frac{\partial \mathcal{W}}{\partial \Lambda} \frac{\partial \overline{\mathcal{W}}}{\partial \Lambda^{\dagger}} \\
& =-\frac{r}{4 \mathcal{R}(r-\mathcal{R})}\left[\left(\partial_{\mu} \mathcal{R}\right)^{2}+\frac{1}{4 \pi^{2}}\left(\partial_{\mu} \Theta\right)^{2}\right]-\frac{|m|^{2}}{r} \mathcal{R}(r-\mathcal{R})
\end{aligned}
$$

where $g^{\Lambda \bar{\Lambda}}=1 / g_{\Lambda \bar{\Lambda}}$. We can immediately compare this with the Lagrangian (2.18) for the bosonic fields $\varphi$ and $\alpha$ and deduce the identifications,

$$
\mathcal{R}=r \sin ^{2} \frac{\varphi}{2} \quad \partial_{\mu} \Theta=\pi r \sin ^{2} \varphi \varepsilon_{\mu \nu} \partial^{\nu} \alpha
$$

The second equality states that the scalar 'field strengths' $\partial_{\mu} \Theta$ and $\partial_{\mu} \alpha$ are related by a twodimensional analog of electric-magnetic duality which interchanges equations of motion and Bianchi identities [11. The bosonic potential,

$$
U=g^{\Lambda \bar{\Lambda}} \frac{\partial \mathcal{W}}{\partial \Lambda} \frac{\partial \overline{\mathcal{W}}}{\partial \Lambda^{\dagger}}=\frac{|m|^{2}}{r} \mathcal{R}(r-\mathcal{R})
$$


has zeros at $\mathcal{R}=0$ and $\mathcal{R}=r$ corresponding to the two supersymmetric vacua of the model. The field $\Theta$ can take any constant value in the vacuum as it only its derivatives appear in the Lagrangian. Note that the zeros of $U$ come from poles in the Kähler metric $g_{\Lambda \bar{\Lambda}}$ rather than zeros of $\partial \mathcal{W} / \partial \Lambda$ which is more conventional. This does not indicate any pathology of the underlying model but rather reflects our choice of coordinates in field space.

We will now consider solitons which interpolate between between distinct vacua at left and right spatial infinity. Specifically we consider boundary conditions, $\Lambda \rightarrow 0$ as $x \rightarrow-\infty$ and $\Lambda \rightarrow \Lambda_{+}=i r+\Delta \Theta / 2 \pi$ as $x \rightarrow+\infty$. The mass of such a configuration obeys the following inequality [16] which hold for any complex constant $\gamma$ with $|\gamma|=1$,

$$
\begin{aligned}
M & =\int_{-\infty}^{+\infty} d x\left[g_{\Lambda \bar{\Lambda}} \frac{\partial \Lambda}{\partial x} \frac{\partial \Lambda^{\dagger}}{\partial x}+g^{\Lambda \bar{\Lambda}} \frac{\partial \mathcal{W}}{\partial \Lambda} \frac{\partial \overline{\mathcal{W}}}{\partial \Lambda^{\dagger}}\right] \\
& =\int_{-\infty}^{+\infty} d x\left|\frac{\partial \Lambda}{\partial x}-\gamma g^{\Lambda \Lambda^{\dagger}} \frac{\partial \overline{\mathcal{W}}}{\partial \Lambda^{\dagger}}\right|^{2}+\int_{-\infty}^{+\infty} d x\left[\bar{\gamma} \frac{\partial \mathcal{W}}{\partial \Lambda} \frac{\partial \Lambda}{\partial x}+\gamma \frac{\partial \overline{\mathcal{W}}}{\partial \Lambda^{\dagger}} \frac{\partial \Lambda^{\dagger}}{\partial x}\right] \\
& \geq \operatorname{Re}\left[\bar{\gamma}\left(\mathcal{W}\left(\Lambda_{+}\right)-\mathcal{W}(0)\right)\right]
\end{aligned}
$$

By choosing $\gamma=\Delta \mathcal{W} /|\Delta \mathcal{W}|$ with $\Delta \mathcal{W}=\mathcal{W}\left(\Lambda_{+}\right)-\mathcal{W}(0)$ we obtain the Bogomol'nyi bound $M \geq 2|\Delta \mathcal{W}|$. This corresponds to a non-zero value for the central charge $Z=2 \Delta \mathcal{W}=i m \Delta \Lambda=$ $-m r+i m \Delta \Theta / 2 \pi$.

To interpret this formula recall that in the $(\theta, \alpha)$ variables we found time-dependent solutions (2.19) with $\alpha=\omega t$. The identification (2.27) implies that time-dependence of $\alpha$ translates to $x$ dependence for $\Theta$. An explicit comparison of soliton solutions in the two sets of variables $(\varphi, \alpha)$ and $(\mathcal{R}, \Theta)$ is performed in Appendix A, where it is shown that the time-dependent dyon solutions (2.19) in the first set of variables correspond to static BPS configurations which saturate the bound (2.29) in the second. In particular the relevant boundary conditions for $\Theta$ are related to the global $U(1)$ charge $S$ as $\Delta \Theta=\theta+2 \pi S$. The resulting BPS mass formula is $M=|Z|$ with central charge $Z=2 \Delta \mathcal{W}=i m(S+\tau)$ which agrees with (2.22) in the sector of topological charge $T=1$. Note that the fact that $S$ is quantized in integer units, which is derived from the Bohr-Sommerfeld quantization condition in the $(\varphi, \alpha)$ variables, is not at all obvious in the $(\mathcal{R}, \Theta)$ variables. The two descriptions are analogous to the two different choices of gauge, due to Tomboulis and Woo (TW) [17] and to Julia and Zee (JZ) [18 respectively, used to describe BPS dyon solutions in four-dimensions. In the TW gauge the dyon solution has periodic time-dependence, while in the JZ gauge it is static. Similarly, the quantization of electric charge is obvious in the first gauge but not in the second.

As emphasised above and in [2], the BPS spectrum of the mass-deformed supersymmetric $C P^{1} \sigma$-model has strong similarities to that of a gauge theory with extended supersymmetry 
in four-dimensions. This correspondence becomes particularly clear in the $(\mathcal{R}, \Theta)$ variables introduced above. To exhibit this connection it is useful to recall another general property of $\mathcal{N}=(2,2)$ solitons in theories of Landau-Ginzburg type. As shown in [6], the soliton always follows a particular trajectory in field space: a straight line in the complex $\mathcal{W}$ plane which joins two vacua. In the present case we have $\mathcal{W}=i m \Lambda / 2$, so this is equivalent to a straight line-segment in the complex $\Lambda$-plane. This is demonstrated explicitly in Appendix A. The mass of the resulting BPS state is proportional to the length of this line-segment. As above we set $\Lambda=i \mathcal{R}+\Theta / 2 \pi$ and note that vacua occur on the two lines $\mathcal{R}=0$ and $\mathcal{R}=r$. Further, it follows from the discussion above that the vacuum values of $\Theta$ are also quantized in units of of $2 \pi S$. They can be chosen to lie at $\Theta=2 \pi n$ for $\mathcal{R}=0$ and $\Theta=2 \pi n+\theta$ for $\mathcal{R}=r$. We now have two infinite rows of vacua in the complex $\Lambda$-plane and each BPS state, including both elementary particles and dyons as well as the corresponding charge-conjugate states, is associated with a line segment joining two vacua. The mass of each state is the length of the line segment. This is essentially identical to the classical charge lattice of BPS states of a four-dimensional $\mathcal{N}=2$ theory|[ with gauge group $S U(2)$, where we identify the quantum numbers $S$ and $T$ correspond to the electric and magnetic charges respectively. Interestingly, the BPS charge lattice which is an abstraction in the four-dimensional context, acquires direct physical significance as a lattice of vacua in field space in the two dimensional theory.

We now turn to the BPS soliton spectrum for the models with general $N$ and $\tilde{N}$. Some partial results for arbitrary $N$ and $\tilde{N}=0$ were given in [2]. In the general case (with $r>0$ ), the central charge of the classical theory is given by,

$$
Z=Z_{S}+Z_{T}=i \sum_{i=1}^{N} m_{i}\left(S_{i}+\tau T_{i}\right)+i \sum_{\tilde{i}=1}^{\tilde{N}} \tilde{m}_{\tilde{i}} \tilde{S}_{\tilde{i}}
$$

In addition to the spectrum of BPS saturated elementary particles described above, there are BPS solitons interpolating between each pair of vacua $\mathcal{V}_{l}$ and $\mathcal{V}_{k}$. The (time-independent) soliton solutions are obtained by a simple embedding of the $C P^{1}$ soliton (2.17) and (2.19) by taking

$$
w_{k}^{(l)}(x)=\exp \pm\left|m_{l}-m_{k}\right| x
$$

where $w_{k}^{(l)}$ is the scalar component of the superfield $W_{k}^{(l)}$ introduced above. This soliton carries topological charge $T_{i}= \pm \delta_{i l} \mp \delta_{i k}$. Each of these solitons yields an infinite tower of dyons which also carry global charges $S_{i}=S T_{i}$ with integer $S$. However, this is not the complete story since the soliton can also form bound-states with the various fermions in the theory. The

\footnotetext{
${ }^{6}$ Recall that only BPS states of magnetic charge $0, \pm 1$ occur in $S U(2) \mathcal{N}=2$ supersymmetric Yang-Mills theory. Each of these states corresponds to a vector joining two vacua in the complex $\Lambda$ plane.
} 
soliton has an associated fermion $\psi_{k}^{(l)}$, the superpartner of $w_{k}^{(l)}$, which possesses two zero-modes in the background of the soliton (2.31), this is true even in the $C P^{1}$ case [2]. These zeromodes are associated to fermionic creation operators which create states that fill out the BPS supermultiplet of the soliton. In the $C P^{1}$ model this is the complete story; however, for the more general models there are more fermion fields and the possibility of more bound-states exists.

In particular, the (time-independent) Dirac equation of the two-component fermion fields $\psi_{j}^{(l)}, j \neq k$, and $\tilde{\psi}_{\tilde{j}}^{(l)}$ is non-trivial in the soliton background:

$$
\left(\begin{array}{cc}
\left|m^{\prime}\right| \sin \alpha & i \partial_{x}+i\left|m^{\prime}\right| \cos \alpha-2 i|m| \rho^{-1} w^{2} \\
i \partial_{x}-i\left|m^{\prime}\right| \cos \alpha & -\left|m^{\prime}\right| \sin \alpha
\end{array}\right) \psi=E \psi
$$

where $\alpha=\arg \left(m^{\prime} / m\right)$. In the above, $w=\exp |m| x$ is the soliton solution, with topological charge $T_{i}=\delta_{i l}-\delta_{i k}, m=m_{l}-m_{k}$ and $\psi$ is one of the fermion fields $\psi_{j}^{(l)}$, or $\tilde{\psi}_{\tilde{j}}^{(l)}$, with $m^{\prime}$ equal to $m_{l}-m_{j}$ and $m_{l}-\tilde{m}_{\tilde{j}}$, respectively. The equation admits a normalizable solution, namely

$$
\psi=\left(\begin{array}{l}
1 \\
0
\end{array}\right) \exp \left(\left|m^{\prime}\right| \cos \alpha x\right)
$$

with energy $E=\left|m^{\prime}\right| \sin \alpha$, as long as

$$
0<\left|m^{\prime}\right| \cos \alpha<|m|, \quad \text { i.e. } \quad 0<\operatorname{Re}\left(\frac{m^{\prime}}{m}\right)<1
$$

In the standard picture of semi-classical quantization, this normalizable mode is then associated with a fermion creation and annihilation operator, $\rho$ and $\rho^{\dagger}$, with the usual anti-commutation relation $\left\{\rho, \rho^{\dagger}\right\}=1$. The soliton carries the two-dimensional Fock space representation of these operators, i.e. it can exist in the vacuum state $|0\rangle$, the original soliton, and the bound-state $\rho^{\dagger}|0\rangle$. Since the fermion mode carries an energy $\left|m^{\prime}\right| \sin \alpha$, the bound-state has a different mass than the soliton $M=|i \tau m|$. However, the bound-state is still a BPS state because the fermion mode contributes to the central charge. The argument goes as follows: the bound-state has central charge $Z=i \tau m+i m^{\prime}$ and therefore to be a BPS state the mass should be

$$
M_{\text {b.s. }}=\left|i \tau m+m^{\prime}\right|=|i \tau m|+\left|m^{\prime}\right| \sin \alpha+\cdots
$$

The first term is the mass of the soliton and the second is the energy of the fermion mode and the higher term are suppressed in $r^{-1}$. So to leading order in $r^{-1}$, the bound-state is indeed a BPS state. The corrections in $r^{-1}$ arise from the back-reaction of the fermion field on the soliton that we have ignored in our leading-order analysis. At the boundaries of the region where the fermion mode becomes non-normalizable, the bound-state will decay into a soliton and a fundamental fermion. 
We can now build up the following picture of the spectrum of topologically charged BPS states. For a soliton of topological charge $T_{i}=\delta_{i l}-\delta_{i k}$, there is tower of dyon states with $S_{i}=S T_{i}$. In addition, for each state of the tower there are bound-state with fermions:

(1) With $\psi_{j}^{(l)}$ in the region

$$
0<\operatorname{Re}\left(\frac{m_{l}-m_{j}}{m_{l}-m_{k}}\right)<1
$$

The bound-state has $S_{i}=S T_{i}+\delta_{i l}-\delta_{i j}$ and $\tilde{S}_{\tilde{i}}=0$. At the boundary $\operatorname{Re}\left(m_{l}-m_{j} / m_{l}-m_{k}\right)=0$ the bound-state decays to the soliton with $S_{i}=S T_{i}$ and the fundamental fermion with $S_{i}=$ $\delta_{i l}-\delta_{i j}$ and $\tilde{S}_{\tilde{i}}=0$, while at the other boundary $\operatorname{Re}\left(m_{l}-m_{j} / m_{l}-m_{k}\right)=1$ the bound-state decays to the soliton with $S_{i}=(S+1) T_{i}$ and the fundamental fermion with $S_{i}=\delta_{i k}-\delta_{i j}$ and $\tilde{S}_{\tilde{i}}=0$.

(2) With $\tilde{\psi}_{\tilde{j}}^{(l)}$ in the region

$$
0<\operatorname{Re}\left(\frac{m_{l}-\tilde{m}_{\tilde{j}}}{m_{l}-m_{k}}\right)<1
$$

The bound-state has $S_{i}=S T_{i}+\delta_{i l}$ and $\tilde{S}_{\tilde{i}}=-\delta_{\tilde{i} \tilde{j}}$. At the boundary $\operatorname{Re}\left(m_{l}-\tilde{m}_{\tilde{j}} / m_{l}-m_{k}\right)=0$ the bound state decays to the soliton with $S_{i}=S T_{i}$ and the fundamental fermion with $S_{i}=\delta_{i l}$ and $\tilde{S}_{\tilde{i}}=-\delta_{\tilde{i} \tilde{j}}$, while at the other boundary $\operatorname{Re}\left(m_{l}-\tilde{m}_{\tilde{j}} / m_{l}-m_{k}\right)=1$ the bound state decays to the soliton with $S_{i}=(S+1) T_{i}$ and the fundamental fermion with $S_{i}=\delta_{i k}$ and $\tilde{S}_{\tilde{i}}=-\delta_{\tilde{i} \tilde{j}}$.

In overlapping regions (2.36) and (2.37), for a number of different fermions $\left\{j_{1}, \ldots, j_{p}, \tilde{j}_{1}, \ldots, \tilde{j}_{s}\right\}$ there will be multiple bound-states carrying global charges

$$
S_{i}=S T_{i}+(p+s) \delta_{i l}-\delta_{i j_{1}}-\cdots-\delta_{i j_{p}}, \quad \tilde{S}_{\tilde{i}}=-\delta_{i \tilde{j}_{1}}-\cdots-\delta_{\tilde{i} \tilde{j}_{s}}
$$

\section{Theory A: Quantum Effects}

In this section we consider how the classical BPS spectrum of Theory A, obtained in the previous section, is modified by quantum corrections. One particularly important quantum effect, which arises at one loop, is the logarithmic running of the of the FI parameter. When all twisted masses are set to zero,

$$
r(\mu)=r_{0}-\frac{(N-\tilde{N})}{4 \pi} \log \left(\frac{M_{U V}^{2}}{\mu^{2}}\right)
$$


where $r(\mu)$ is the renormalized FI parameter, defined at the scale $\mu$, and $M_{U V}$ is a UV cut-off. The bare FI parameter, $r_{0}$, is equal to the renormalized FI parameter, $r(\mu)$, evaluated at the cut-off scale $\mu=M_{U V}$. In the limit $e \rightarrow \infty$, the theory reduces to a supersymmetric $\sigma$-model with target space $\mathcal{M}_{H}$ as described in the previous section. The running coupling of the $\sigma$ model is related to the FI parameter as $g(\mu)=\sqrt{2 / r(\mu)}$. We will assume that $r_{0} \gg 0$ so that the resulting $\sigma$-model is weakly-coupled at the cutoff scale. As usual the running coupling may be eliminated in favour of an RG invariant scale defined by,

$$
\Lambda=\mu \exp \left(-\frac{2 \pi r(\mu)}{N-\tilde{N}}\right)
$$

The sign of the $\sigma$-model $\beta$-function depends on the $\operatorname{sign}$ of $N-\tilde{N}$. If $N>\tilde{N}$ then the theory is asymptotically free and, in the absence of twisted masses, will run to strong coupling at low energies. This, for example, is the behaviour of the supersymmetric $C P^{N-1} \sigma$-model. As explained in [2], this behaviour is modified when non-zero twisted masses are introduced. The running of the coupling is driven by quantum fluctuations of the light chiral multiplets which appear in the $\sigma$-model Lagrangian. All these fields decouple at energy scales below some scale $M$ which is roughly the twisted mass of the lightest chiral multiplet. The running coupling is therefore frozen below this energy scale. Provided we choose twisted masses such that $\left|m_{i}-m_{j}\right| \gg \Lambda$ for enough pairs $i$ and $j$, then the resulting $\sigma$-model will be weakly coupled at all energies. On the other hand, if $N<\tilde{N}$, then the $\sigma$-model is IR free. Such a theory may however suffer from a Landau pole in the UV, and should usually only be considered as a low-energy effective theory valid below the mass scale $\Lambda$ which may be much lower than $M_{U V}$. Finally, the massless theory with $N=\tilde{N}$ has vanishing beta-function and is therefore scale invariant. We will henceforth restrict our attention to the asymptotically free case $N>\tilde{N}$ unless otherwise stated.

Another important effect which arises at one-loop is an anomaly which breaks the $U(1)_{A}$ R-symmetry down to $Z_{2 N-2 \tilde{N}}$. In the absence of twisted masses this means that the bare $\theta$-parameter can be set to zero by a $U(1)_{A}$ rotation of the fields. However, even if the bare parameter is set to zero the theory will still have an effective vacuum angle at low energy. If the twisted masses are non-zero then the $U(1)_{A}$ symmetry is already explicitly broken to $Z_{2}$ at the classical level. As discussed in [2], the one-loop effects of the running coupling and the anomaly are equivalent to a holomorphic renormalization of the complex coupling $\tau$. In the $C P^{1}$ case described in the previous section this amounts to replacing $\tau$ by,

$$
\tau_{\text {eff }}=i r_{\text {eff }}+\theta_{\text {eff }} / 2 \pi=\frac{i}{\pi} \log \left(\frac{m}{\Lambda}\right)
$$

When this replacement is combined with the $\theta$-dependent shift in global $U(1)$ charge of the dyons described above, it leads to a non-trivial monodromy in the dyon spectrum which is a 
close analog of the weak-coupling monodromy of the BPS spectrum of an $\mathcal{N}=2$ theory in four dimensions.

So far we have only discussed the spectrum in the IR limit $e \rightarrow \infty$ when the gauge theory we started with reduces to a non-linear $\sigma$-model. As long as the twisted masses are large and the $\sigma$-model is weakly coupled, this limit is convenient for determining the BPS spectrum. However for more general values of the twisted masses, the $\sigma$-model becomes strongly coupled and a new approach is required. A key property of theories with $\mathcal{N}=(2,2)$ supersymmetry in two dimensions is that the masses of BPS states are effectively determined by the F-terms in the superspace Lagrangian. As the two dimensional gauge coupling $e$ only appears in the gauge multiplet kinetic term, which is a D-term, it follows that the masses of BPS states are actually independent of $e$. This suggests a completely different regime in which we may attempt to determine the BPS spectrum. When $e$ is much less than the other mass scales in the problem we should be able to determine the BPS spectrum using ordinary perturbation theory. Note that perturbation theory in the two-dimensional gauge coupling is quite distinct from the perturbation theory in the $\sigma$-model coupling used above.

Following [10], we consider the effective Lagrangian along the 'Coulomb branch' $\phi_{i}=\tilde{\phi}_{\tilde{i}}=0$ with $\sigma$ unconstrained. As discussed in the previous section, the are no classical SUSY vacua on this branch unless $r=0$. However, even at weak coupling, this conclusion can be altered by quantum effects. As long as $e$ is much less than each of the scales $\left|\sigma+m_{i}\right|,\left|\sigma+\tilde{m}_{\tilde{i}}\right|$ and $\Lambda$, we have a theory of a light $U(1)$ gauge multiplet weakly coupled to massive chiral multiplets. In this case we may integrate out the chiral multiplets and get an effective Lagrangian for the gauge degrees of freedom of the form,

$$
\mathcal{L}_{\text {eff }}=\int d^{4} \vartheta \mathcal{K}_{\text {eff }}\left[\Sigma, \Sigma^{\dagger}\right]+\int d^{2} \vartheta \mathcal{W}_{\text {eff }}[\Sigma]+\int d^{2} \bar{\vartheta} \overline{\mathcal{W}}_{\text {eff }}\left[\Sigma^{\dagger}\right]
$$

A one-loop calculation of the effective twisted superpotential yields [1],

$$
\mathcal{W}_{\text {eff }}=\frac{i}{2} \hat{\tau} \Sigma-\frac{1}{4 \pi} \sum_{i=1}^{N}\left(\Sigma+m_{i}\right) \log \left(\frac{2}{\mu}\left(\Sigma+m_{i}\right)\right)+\frac{1}{4 \pi} \sum_{\tilde{i}=1}^{\tilde{N}}\left(\Sigma+\tilde{m}_{\tilde{i}}\right) \log \left(-\frac{2}{\mu}\left(\Sigma+\tilde{m}_{\tilde{i}}\right)\right)
$$

Here, the complexified coupling constant $\hat{\tau}$ is equal to $\operatorname{ir}(\mu)+\theta / 2 \pi+n^{*}$ where the integer $n^{*}$ is chosen to minimize the potential energy. As explained in [10], this minimization of the potential reflects the fact that a non-zero value of the $\theta$ parameter in two-dimensions corresponds to a constant background electric field [19]. The states of the system with $n \neq n^{*}$, are unstable to pair creation of charged particles which screens the background field leaving the state with $N=n^{*}$. In fact there are various arguments which suggest that this is the exact twisted superpotential [20, 6]. In any case, we will always take $e$ sufficiently small so that the twisted 
superpotential is reliable. In general the only points where this effective description may break down even at small $e$ are the points $\sigma=-m_{i}$ and $\sigma=-\tilde{m}_{\tilde{i}}$ where we will find that extra light degrees of freedom must be included.

The potential energy of the effective theory is,

$$
U=g^{\Sigma \bar{\Sigma}}\left|\frac{\partial \mathcal{W}_{\mathrm{eff}}}{\partial \Sigma}\right|^{2}
$$

where $g^{\Sigma \bar{\Sigma}}=\left(g_{\Sigma \bar{\Sigma}}\right)^{-1}$ is the inverse of the Kähler metric,

$$
g_{\Sigma \bar{\Sigma}}=-\frac{\partial^{2} \mathcal{K}_{\mathrm{eff}}}{\partial \Sigma \partial \Sigma^{\dagger}}
$$

At weak coupling, corrections to the tree level Kähler potential $\mathcal{K}_{\text {tree }}=\Sigma^{\dagger} \Sigma / 4 e^{2}$, are small an we can safely assume that the metric does not have poles or zeros in the region of field space where our approximations are valid. Thus the supersymmetric vacua of the theory are in one to one correspondence with the zeros of $\partial \mathcal{W} / \partial \Sigma$ and are therefore determined by the complex equation,

$$
\frac{\prod_{i=1}^{N}\left(\sigma+m_{i}\right)}{\prod_{\tilde{i}=1}^{\tilde{N}}\left(\sigma+\tilde{m}_{\tilde{i}}\right)}=\tilde{\Lambda}^{N-\tilde{N}}
$$

where $\tilde{\Lambda}=\frac{1}{2}(-1)^{\tilde{N} /(N-\tilde{N})} \Lambda \exp (-1+i \theta /(N-\tilde{N}))$. Provided the numerator and denominator on the LHS of (3.8) do not have common zeros, the equation becomes,

$$
\prod_{i=1}^{N}\left(\sigma+m_{i}\right)-\tilde{\Lambda}^{N-\tilde{N}} \prod_{\tilde{i}=1}^{\tilde{N}}\left(\sigma+\tilde{m}_{\tilde{i}}\right)=\prod_{i=1}^{N}\left(\sigma-e_{i}\right)=0
$$

Thus there are $N$ supersymmetric vacua located at $\sigma=e_{i}$ for $i=1, \ldots, N$. As above, we have assumed that $N>\tilde{N}$. For $\left|m_{i}-m_{j}\right| \gg \Lambda$, these vacua coincide with the $N$ classical vacua $\mathcal{V}_{i}$, located at the points $\sigma=-m_{i}$, defined in the previous section.

We now consider a BPS saturated soliton obeying the boundary conditions, $\sigma \rightarrow e_{k}$ as $x \rightarrow-\infty$ and $\sigma \rightarrow e_{l}$ as $x \rightarrow+\infty$. As the effective Lagrangian is of the standard LandauGinzburg form discussed in the previous section we may apply the general BPS mass formula obtained there. Thus, the soliton mass is given by $M_{k l}=\left|Z_{k l}\right|$ where $Z_{k l}=2 \Delta \mathcal{W}_{\text {eff }}=2 \mathcal{W}_{\text {eff }}\left(e_{l}\right)-$ $2 \mathcal{W}_{\text {eff }}\left(e_{k}\right)$. A short calculation reveals that,

$$
Z_{k l}=\frac{1}{2 \pi}\left[(N-\tilde{N})\left(e_{l}-e_{k}\right)-\sum_{i=1}^{N} m_{i} \log \left(\frac{e_{l}+m_{i}}{e_{k}+m_{i}}\right)+\sum_{\tilde{i}=1}^{\tilde{N}} \tilde{m}_{\tilde{i}} \log \left(\frac{e_{l}+\tilde{m}_{\tilde{i}}}{e_{k}+\tilde{m}_{\tilde{i}}}\right)\right]
$$


For non-zero twisted masses, the branch-cuts in the logarithms appearing in (3.10) lead to an ambiguity in the BPS spectrum. In particular the ambiguity in the central charge is equal to $i \sum_{i=1}^{N} m_{i} n_{i}+i \sum_{\tilde{i}=1}^{\tilde{N}} \tilde{m}_{\tilde{i}} \tilde{n}_{\tilde{i}}$ where the choice of integers $n_{i}$ and $\tilde{n}_{\tilde{i}}$ corresponds to a choice of branch for each of the $N+\tilde{N}$ logarithms in (3.10). As explained by Hanany and Hori in [1], this ambiguity signals the fact that solitons can carry integer values of the global $U(1)$ charges $S_{i}, \tilde{S}_{\tilde{i}}$ in addition to their topological charges. This is related to the existence of BPS dyons at weak-coupling discussed in the previous sections. Including this effect, the final formula for the masses of all BPS states in the theory is $M=|Z|$ with, charge is

$$
Z=i \sum_{i=1}^{N}\left(m_{i} S_{i}+m_{D i} T_{i}\right)+i \sum_{\tilde{i}=1}^{\tilde{N}} \tilde{m}_{\tilde{i}} \tilde{S}_{\tilde{i}}
$$

which differs from the classical formula (2.30) of the previous section by the replacement of $m_{D i}^{c l}=\tau m_{i}$ by

$$
m_{D i}=-2 i \mathcal{W}_{\text {eff }}\left(e_{i}\right)=\frac{1}{2 \pi i}\left[(N-\tilde{N}) e_{i}-\sum_{j=1}^{N} m_{j} \log \left(e_{i}+m_{j}\right)+\sum_{\tilde{j}=1}^{\tilde{N}} \tilde{m}_{\tilde{j}} \log \left(e_{i}+\tilde{m}_{\tilde{j}}\right)\right]
$$

For $\left|m_{i}-m_{j}\right| \gg \Lambda$, this formula can be directly compared with the results of the semiclassical analysis given in the previous sections. In this limit, the replacement $\tau \rightarrow \tau_{\text {eff }}=\partial \mathcal{W} / \partial \sigma$ reproduces the one-loop holomorphic renormalization of $\tau$, given above by $(3.3)$ in the $C P^{1}$ case, while in the general case the semi-classical limit is

$$
m_{D i} \rightarrow \frac{1}{2 \pi i}\left[-(N-\tilde{N}) m_{i}-\sum_{j=1}^{N}\left(m_{j}-m_{i}\right) \log \left(\frac{m_{j}-m_{i}}{\tilde{\Lambda}}\right)+\sum_{\tilde{j}=1}^{\tilde{N}}\left(\tilde{m}_{\tilde{j}}-m_{i}\right) \log \left(\frac{\tilde{m}_{\tilde{j}}-m_{i}}{\tilde{\Lambda}}\right)\right]
$$

For particular values of the twisted masses there are various singular points which can be compared with the corresponding singular points in the classical theory. For example, in the previous section we discovered that if two twisted masses $m_{i}$ and $m_{j}$ coincide in the classical theory, then the chiral multiplets $\phi_{i}$ and $\phi_{j}$ are both massless at $\sigma=-m_{1}=-m_{2}$ giving a Higgs branch which is a copy of $C P^{1}$. As the resulting low-energy $\sigma$-model is strongly coupled in the IR we should expect that quantum effects modify this singularity. As explained in [2], the classical singular point $m=m_{i}-m_{j}=0$, is split into a pair of singular points at which the vacua $e_{i}$ and $e_{j}$ coincide. At each of these singular points a single chiral multiplet, corresponding to the soliton which interpolates between the vacua $\mathcal{V}_{i}$ and $\mathcal{V}_{j}$, becomes massless. A single massless chiral multiplet does not yield a continuous Higgs branch and, in particular, does not result in a massless Goldstone boson. Thus there is no conflict with the usual restrictions on massless particles in two dimensions [13]. 
In the present case we can also consider what happens if chiral multiplets of opposite charge become massless. In the classical theory this happens if $m_{i}=\tilde{m}_{\tilde{j}}$ for some $i$ and $\tilde{j}$. As in the previous case this gives a Higgs branch of complex dimension one at $\sigma=-m_{i}=-\tilde{m}_{\tilde{j}}$. However the present Higgs branch has zero first Chern class and the corresponding low-energy $\sigma$-model is scale-invariant. In this case we might reasonably expect the Higgs branch to remain in the full quantum theory. The massless theory on the Higgs branch could either be a free theory or, perhaps, a non-trivial CFT】. In equation (3.8), which determines the exact location of the Coulomb branch vacua, something interesting happens whenever $m_{i}=\tilde{m}_{\tilde{j}}$ for some $i$ and $\tilde{j}$ : the numerator and denominator have a common zero and a cancellation occurs. The degree of the polynomial (3.9) is then lowered by one and it appears that one of the supersymmetric vacua has disappeared. If this were true it would, certainly contradict standard properties of the Witten index. The resolution is simply that, as in the classical theory, a non-compact Higgs branch appears at $\sigma=-m_{i}$. The effective FI parameter on this branch is determined by the twisted superpotential,

$$
\tau_{\mathrm{eff}}=\left.\frac{\partial \mathcal{W}}{\partial \sigma}\right|_{\sigma=-m_{i}}
$$

It is also useful to note that, as the two massless chiral multiplets $\Phi_{i}$ and $\tilde{\Phi}_{j}$ have opposite charges, we can introduce a complex mass $\hat{m}_{i \tilde{j}}$ by including a superpotential of the form (2.2). This lifts the Higgs branch completely and, in this case, we are left with only $N-1$ Coulomb branch vacua. This is particularly clear in the IIA brane picture described in Section 5. A contradiction with the Witten index is avoided because the transition from theory with $N$ vacua to one with $N-1$, always goes via the singular point in parameter space where $m_{i}=\tilde{m}_{\tilde{j}}$, $\hat{m}_{i \tilde{j}}=0$. At this point we have flat directions on the Higgs branch and the Witten index is not defined.

The masses of topologically non-trivial BPS states are patently not single valued as one tracks their mass, as a function of the parameters $m_{i}$ and $\tilde{m}_{\tilde{i}}$, around one of the singularities described above: there is non-trivial monodromy. To make this concrete we can choose a particular branch of the multi-valued functions (3.13) and then interpret the monodromy as transformations on the charges as one crosses a cut. Around the singularity $m_{j}=m_{l}$ the transformation is

$$
T_{i} \rightarrow T_{i}, \quad S_{i} \rightarrow S_{i}+\delta_{i l}-\delta_{i j}, \quad \tilde{S}_{\tilde{i}} \rightarrow \tilde{S}_{\tilde{i}}
$$

while around the second kind of singularity $\tilde{m}_{\tilde{j}}=m_{l}$

$$
T_{i} \rightarrow T_{i}, \quad S_{i} \rightarrow S_{i}+\delta_{i l}, \quad \tilde{S}_{\tilde{i}} \rightarrow \tilde{S}_{\tilde{i}}-\delta_{\tilde{i} \tilde{j}}
$$

\footnotetext{
${ }^{7}$ Neither of these possibilities is in conflict with the absence of Goldstone bosons in two-dimensions.
} 
It is easy to see that if $j \neq k$ in (3.15) and for any $\tilde{j}$ in (3.16), repeated application of these transformations does not preserve the semi-classical spectrum. For instance, for the states with $T_{i}=\delta_{i l}-\delta_{i k}$ and $S_{i}=S T_{i}$, for integer $S$, the monodromy transformation around the singularity $m_{j}=m_{l}, j \neq k, l$, induces $S_{i} \rightarrow S T_{i}+\delta_{i l}-\delta_{i j}$, which is a state in the spectrum in the region (2.36). However, a further such transformation gives $S_{i} \rightarrow S T_{i}+2 \delta_{i l}-2 \delta_{i j}$ which is not commensurate with the semi-classical spectrum. The resolution of this paradox, is that the contour around the singularity $m_{j}=m_{l}$ passes through a curve of marginal stability on which the state decays into two other topologically charged states with charges $T_{i}^{(1)}=\delta_{i l}-\delta_{i j}$, $S_{i}^{(1)}=(S+2) T_{i}^{(1)}$ and $T_{i}^{(2)}=\delta_{i j}-\delta_{i k}, S_{i}^{(2)}=S T_{i}^{(2)}$. Notice that this kind of decay is in addition to the soliton decay to soliton plus fundamental fermion that we saw in the last section. If we think of the masses $m_{i}$ and $\tilde{m}_{\tilde{i}}$ as a set of points in the complex plane, then the curve of marginal stability is identified with configurations of points where $m_{j}$ lies on the straight line joining $m_{k}$ and $m_{l}$. On the curve, the simple semi-classical analysis of section 2 must be re-evaluated because the moduli space of the charge $T_{i}=\delta_{i l}-\delta_{i k}$ soliton is larger than that of the embedded $C P^{1}$ soliton. The enlarged moduli space of solutions, can be interpreted as two superposed solitons with charges $T_{i}^{(1)}$ and $T_{i}^{(2)}$, above, and the original soliton decays at threshold into these two constituents. This mechanism for decay is precisely the same as that seen for dyons at weak coupling in supersymmetric gauge theories [23, 24].

So far we have determined the exact mass formula for BPS states with arbitrary global and topological charges. However, except in the weak-coupling limit of the low-energy $\sigma$-model, we do not know which states are present in the theory. In general, as we vary the parameters appearing in the twisted superpotential, we may cross curves of marginal stability on which BPS states can decay. Even in the simplest case of target space $C P^{1}$, the existence of such curves was demonstrated in [2]. Fortunately there is a special point in parameter space where we may determine the exact BPS spectrum. We first consider the case $\tilde{N}=0$. If we set all twisted masses to zero, we have the standard supersymmetric $C P^{N-1} \sigma$-model. This theory is integrable and its exact spectrum and S-matrix are known. Also, at this point only, the model has an unbroken $S U(N)$ global symmetry and the BPS states are organised in multiplets of this symmetry. To make contact with the analysis of the twisted superpotential we note that, for $\tilde{N}=0$ and $m_{i}=0$ the complex equation (3.8) reduces to $\sigma^{N}=\tilde{\Lambda}^{N}$. The vacua $e_{k}$, $k=1,2, \ldots, N$ are then located at the vertices of a regular $N$-gon in the complex $\sigma$-plane,

$$
e_{k}=\tilde{\Lambda} \exp \left(\frac{2 \pi i k}{N}\right)
$$

As the twisted masses vanish, the theory has a $Z_{2 N}$ symmetry which is spontaneously broken to $Z_{2}$ by the VEV of $\sigma$. The $Z_{N}$ symmetry of the $N$-gon comes from the quotient $Z_{2 N} / Z_{2}$.

According to (3.10), the mass of a soliton interpolating between the vacua with $\sigma=e_{l}$ and 
$\sigma=e_{k}$ is $M_{k, l}=\left|2 \Delta \mathcal{W}_{\text {eff }}\right|$. Because of the $Z_{N}$ symmetry this only depends on the difference $p=l-k$. The resulting mass is

$$
M_{k, l}=\frac{N}{\pi}\left|\exp \left(\frac{2 \pi i p}{N}\right)-1\right| \tilde{\Lambda}
$$

It is also known [6] that for $p=1,2 \ldots N$ the degeneracy of BPS states is,

$$
D_{p}=\left(\begin{array}{c}
N \\
p
\end{array}\right)
$$

The lightest soliton states with $p=1$ and degeneracy $N$ are interpreted as the elementary quanta of the the chiral fields $\Phi_{i}$ [21]. In fact they carry charge +1 under the unbroken $U(1)$ gauge symmetry. These states transform in the fundamental representation of the flavour symmetry group $S U(N)$. The states with $p>1$ correspond to stable bound states of $p$ different flavours of elementary quanta and therefore transform in the $p$ 'th antisymmetric tensor representation of $S U(N)$ which agrees with the degeneracy $D_{p}$ in (3.19). These are the only BPS states of the model. The complete BPS spectrum of the supersymmetric $C P^{N-1} \sigma$-model obtained in this way, including both the fundamental solitons and their boundstates, is consistent with various exact results which can be obtained by invoking the integrability of the model.

In the vicinity of the strong coupling point, where all the masses $m_{i}$ are small, the degeneracy of the states is broken and the states with a topological charge $T_{i}=\delta_{i l}-\delta_{i k}$ are associated to $D_{p}$ different contours in the $\sigma$-plane which join the points $e_{l}$ and $e_{k}$. As explained in [1], the different contours can be constructed from some base contour joining $e_{l}$ and $e_{k}$, by taking the contours which differ from this by encircling, in a positive sense, the $p$ distinct points $m_{j_{a}}$, $a=1, \ldots, p$, chosen from the set $\left\{m_{i}\right\}$. This gives rise to precisely $D_{p}$ different states with global charge

$$
S_{i}=N_{i}-\delta_{i j_{1}}-\cdots-\delta_{i j_{p}}
$$

where $N_{i}$ is some fixed set of integers which depends upon the choice of branch for the multivalued function $m_{D i}$. This preferred set of contours can be shown to arise very naturally from the Type IIA string description of the model [1].

We can now compare the strong coupling and weak coupling spectra. It is clear that, as in the $C P^{1}$ case, there are an a infinite number of states at weak coupling while the set of states at strong coupling is finite. This implies that semi-classical regime must be separated from the strong coupling regime by curves on which almost all of the weak coupling states decay. This is familiar from the behaviour of dyon states in $S U(2) \mathcal{N}=2$ SQCD [7]. Moreover, as in gauge theory case, the strong coupling states are a subset of the weak coupling states since states 
with global charge (3.20) are realized in some region of the moduli space at weak coupling, as bound-states of the soliton with fermions (2.38).

Finally, we will consider the theory without twisted masses in the more general case of arbitrary $N$ and $\tilde{N}$. In this case there is a cancellation of a factor of $\sigma^{\tilde{N}}$ between the numerator and denominator of (3.8). As discussed above this indicates the presence of a non-compact Higgs branch at $\sigma=0$. As $\tilde{N}$ chiral multiplets of each charge become massless at this point the Higgs branch has complex dimension $2 \tilde{N}-1$. After the cancellation the equation which determines the supersymmetric vacua reduces to $\sigma^{N-\tilde{N}}=\tilde{\Lambda}^{N-\tilde{N}}$. This is the same equation which arises in the case of the supersymmetric $C P^{N-\tilde{N}-1} \sigma$-model with zero twisted masses. This is not a coincidence; we can lift the Higgs branch by introducing suitable complex mass terms. As complex masses can be thought of as the scalar components of background chiral superfields they cannot modify the twisted superpotential which determines the soliton masses. By taking the complex masses large we can decouple the $\tilde{N}$ chiral multiplets whose VEVs parametrize the Higgs branch and the resulting theory theory has $N-\tilde{N}$ chiral multiplets of charge +1 under $U(1)_{G}$ and none of charge -1 . The theory then reduces to the supersymmetric $C P^{N-\tilde{N}} \sigma$-model at low-energy. As for the $\tilde{N}=0$ models discussed above, the strong coupling spectrum contains a subset of the states that appear at weak coupling.

We will now briefly summarize our results for Theory A. The exact central charge of the theory is defined by (3.11) and (3.12). This formula is valid for all $e$ and it applies equally in the $\sigma$-model limit $e \rightarrow \infty$. If enough of the twisted mass differences $\left|m_{i}-m_{j}\right|$ are much greater than $|\Lambda|$, the low-energy $\sigma$-model is weakly coupled and the BPS spectrum is essentially that of the classical theory described in the previous section. In particular the BPS spectrum in the region consists of elementary particles, kinks which interpolate between each pair of vacua, and an infinite tower of dyons associated with each kink. There are also soliton-fermion boundstates. On the other hand we have also determined the BPS spectrum for the theory with zero twisted masses. In this case we find, $2 \tilde{N}-1$ massless chiral multiplets on the Higgs branch while on the Coulomb branch there are $N-\tilde{N}$ supersymmetric vacua and a spectrum of massive solitons identical to that of the supersymmetric $C P^{N-\tilde{N}-1}$ model. The model with zero twisted masses has a global $S U(N-\tilde{N}) \times U(\tilde{N})$ symmetry and non-anomalous $Z_{2 N-2 \tilde{N}}$ R-symmetry which is spontaneously broken to $Z_{2}$. Introducing complex masses lifts the Higgs branch but does not change the Coulomb branch vacua or the BPS soliton mass. On the other hand, introducing non-zero twisted masses breaks the global $S U(N-\tilde{N})$ symmetry. If the masses are small the only effect is to introduce small mass splittings in the multiplets described above. However, as the masses are increased the theory can cross curves of marginal stability on which the BPS spectrum can change. In fact, the presence of such curves is certainly required to explain the difference between the strong coupling spectrum obtained here which has a finite number of 
BPS states and the weak coupling spectrum which has an infinite number.

\section{Theory B}

We turn now to the spectra of the four-dimensional theories described in the introduction. Hence we will consider $\mathcal{N}=2 \mathrm{SQCD}$ with $N$ colours and $N_{f}>N$ fundamental hypermultiplets with masses $m_{\lambda}$ for $\lambda=1, \ldots, N_{f}$. This theory has received a great deal of attention in recent years and we need only be brief. The bosonic field content of Theory B consists of an $S U(N)$ gauge field and a single complex scalar, $\phi$, transforming in the adjoint representation of the gauge group. A further $N_{f}$ complex scalars, denoted $Q_{a}^{\lambda}$, with $a=1, \ldots, N$, transform in the fundamental representation of the gauge group and $N_{f}$ complex scalars, $\tilde{Q}_{\lambda}^{a}$, in the antifundamental representation.

The moduli space of supersymmetric vacua is found by solving the classical D- and Fterm equations. A detailed description of the various branches which occur was given in [4] and we will now summarize the relevant results. The classical theory has a Coulomb branch on which the adjoint scalar VEV lies in the Cartan subalgebra of the gauge group: $\phi=$ $\operatorname{diag}\left(\phi_{1}, \ldots, \phi_{N}\right)$ with $\sum_{a=1}^{N} \phi_{a}=0$. The complex variables $\phi_{a}$, with $a=1, \ldots, N$, parametrize gauge-inequivalent vacua, modulo the Weyl group of $S U(N)$. The theory also has various Higgs branches which are characterised as 'baryonic' or 'non-baryonic', depending on whether or not there is a non-vanishing $\mathrm{VEV}$ of the gauge invariant operators,

$$
\begin{aligned}
B^{\lambda_{1} \ldots \lambda_{N}} & =Q_{a_{1}}^{\lambda_{1}} \ldots Q_{a_{N}}^{\lambda_{N}} \epsilon^{a_{1} \ldots a_{N}} \\
\tilde{B}_{\lambda_{1} \ldots \lambda_{N}} & =\tilde{Q}_{\lambda_{1}}^{a_{1}} \ldots \tilde{Q}_{\lambda_{N}}^{a_{N}} \epsilon_{a_{1} \ldots a_{N}}
\end{aligned}
$$

Non-baryonic branches have $B=\tilde{B}=0$ and are parametrized instead by the gauge-invariant 'mesons' $M_{\rho}^{\lambda}=Q_{\rho}^{a} \tilde{Q}_{a}^{\lambda}$. The classical Coulomb branch is corrected by quantum effects while the Higgs branches, being protected by a non-renormalization theorem, are not. The Higgs branches intersect the Coulomb branch at singular points where extra degrees of freedom become massless. Although the Higgs branches themselves are not corrected, their intersection with the Coulomb branch may be different in the classical and quantum theories.

In the present context we are interested in the theory at the root of its first baryonic branch. In the classical theory with vanishing masses, $m_{\lambda}=0$, this branch has quaternionic dimension $N_{f} N-N^{2}+1$ and emanates from the origin of the Coulomb branch. When non-zero masses are introduced we must solve the modified F-term equations,

$$
Q_{a}^{\lambda}\left(m_{\lambda}+\phi_{a}\right)=\tilde{Q}_{\lambda}^{a}\left(m_{\lambda}+\phi_{a}\right)=0 \quad \text { No summation over } \lambda \text { or } a .
$$


In order for the scalars to satisfy (4.1) while still having non-vanishing expectation value for $B$ and $\tilde{B}$, the adjoint scalar VEVs must take values $\phi_{a}=-m_{\lambda(a)}$, where $\left\{m_{\lambda(a)}\right\}$ is a set of $N$ masses chosen from the $m_{\lambda}, \lambda=1, \ldots, N_{f}$. Up to Weyl group transformations, there are $N_{f}$ choose $N$ possible sets and each choice gives a baryonic root from which a Higgs branch of complex dimension one may emanate. To choose a particular root, we will label the masses as $m_{\lambda}=m_{i}$ for $\lambda=i=1, \ldots, N$ and $m_{\lambda}=\tilde{m}_{\tilde{i}}$ with $\lambda=\tilde{i}+N$ for $\tilde{i}=1, \ldots, \tilde{N}=N_{f}-N$ and then set $\phi_{a}=-m_{i} \delta_{i a}$. This solution is only consistent with the tracelessness of the adjoint representation of $S U(N)$ if we have $\sum_{i=1}^{N} m_{i}=0$. As discussed in the introduction the masses $m_{i}$ and $\tilde{m}_{\tilde{i}}$ will be identified with the twisted masses of Theory A which carry the same indices.

At a generic point on its Coulomb branch, the four-dimensional theory has classical central charge,

$$
Z=\sum_{a=1}^{N} \phi_{a}\left(q_{a}+\tau h_{a}\right)+\sum_{\lambda=1}^{N_{f}} m_{\lambda} s_{\lambda}
$$

where $\tau=4 \pi i / g^{2}+\theta / 2 \pi$ is the complexified gauge coupling and $q_{a}, h_{a}$ and $s_{\lambda}$ are integer valued electric, magnetic and global charges respectively. Specializing this expression to the baryonic root defined above we may rewrite the central charge as,

$$
Z=\sum_{i=1}^{N} m_{i}\left(S_{i}+\tau T_{i}\right)+\sum_{\tilde{i}=1}^{\tilde{N}} \tilde{m}_{i} \tilde{S}_{\tilde{i}}
$$

where we have redefined charges as $S_{i}=-s_{a}-q_{a}$ and $T_{i}=-h_{a}$ for $i=a=1, \ldots, N$ and $\tilde{S}_{\tilde{i}}=-s_{\tilde{i}+N}$ for $\tilde{i}=1, \ldots, \tilde{N}$. Note that the electric charges become parallel to a subset of the global charges at the baryonic root and we have absorbed them with an integer shift in the definition of the global generators. The resulting expression for the classical central charge exactly matches its two-dimensional counterpart (2.30)

Let us now consider which values of the charges $S_{i}, \tilde{S}_{i}$ and $T_{i}$ occur in the four dimensional theory. We will consider $\mathcal{N}=2$ vector multiplets and hypermultiplets in turn. At a generic point on the Coulomb branch the theory includes $N-1$ massless abelian vector multiplets which correspond to the unbroken $U(1)^{N-1}$ gauge symmetry. The remaining generators of the gauge group give $N(N-1)$ massive vector multiplets which carry non-zero electric charges. At the baryonic root the charges which arise correspond to $S_{i}=\delta_{i k}-\delta_{i j}$ for each $k \neq j$ Thus we have $N(N-1)$ vector multiplets with distinct masses $\left|m_{k}-m_{j}\right|$. The spectrum at the baryonic root also contains $N$ massless quark hypermultiplets whose scalar components are $Q_{a=i}^{\lambda=i}$ and $\tilde{Q}_{a=i}^{\lambda=i}$ for $i=1, \ldots N$. These states have non-zero electric charges $q_{a=i}$ and non-zero global charges $s_{\lambda=i}$ which cancel to give $S_{i}=0$. These massless quarks each acquire non-zero VEVs on the baryonic branch itself. 
Finally, and most importantly in the present context, Theory B also has a diverse spectrum of massive hypermultiplets which includes quarks, monopoles and dyons. These massive quark states correspond to the remaining components of the hypermultiplets which carry electric charges $q_{a=i}$ and global charges $s_{\lambda=i}$ and yield non-zero values of $S_{i}$ and $\tilde{S}_{\tilde{i}}$. The resulting spectrum includes $N(N-1)$ states with charges $S_{i}=\delta_{i k}-\delta_{i j}$ and $\tilde{S}_{\tilde{i}}=0$ which have masses $\left|m_{k}-m_{j}\right|$ as well as $N\left(N_{f}-N\right)$ states with charges $S_{i}=\delta_{i k}$ and $\tilde{S}_{\tilde{i}}=-\delta_{\tilde{i} \tilde{j}}$ which have masses $\left|m_{k}-\tilde{m}_{\tilde{\tilde{j}}}\right|$. The total number of massive quark hypermultiplets is therefore $N\left(N_{f}-1\right)=$ $N(N+\tilde{N}-1)$. This agrees with the classical spectrum of elementary particles of Theory A.

All further BPS states have non-zero magnetic charge. While the existence of classical solutions in various topological sectors is well known, the existence of quantum bound states is more problematic. For systems with a single Higgs field or, equivalently, for real adjoint Higgs VEVs, $\phi_{a}$, this requires detailed knowledge of the monopole moduli spaces for higher rank gauge groups (see for example [22]). However, as first discussed in [23], the situation simplifies greatly for generic complex VEVs and semi-classical quantization reduces to the corresponding problem with all fields restricted to $S U(2)$ subgroups of the gauge group. The spectrum arising from each subgroup can then be determined using a variety of methods [25, 26, []. The resulting monopole spectrum contains $N(N-1)$ different states with magnetic charges $T_{i}=\delta_{i l}-\delta_{i k}$ for each $l \neq k$ and with masses $4 \pi\left|m_{l}-m_{k}\right| / g^{2}$, in agreement with the classical soliton spectrum of Theory A. In addition to these purely magnetic states, the spectrum also contains an infinite tower of dyons associated to each of the $S U(2)$ subgroups and with charges $S_{i}=S T_{i}$ for integer values of $S$. Once again, this coincides with the excited soliton spectrum of Theory A.

However, just as for Theory A, this is not the whole story because dyons can form boundstates with quarks. To our knowledge, the spectrum of such states has not been constructed in the literature and so to this we devote Appendix B. The results are as follows. We work at a generic point in the moduli space, so that the moduli space of a monopole is that of a monopole in an $S U(2)$ gauge theory. At the baryonic root, we find the following spectrum of bound-states of quarks with the dyon having charges $T_{i}=\delta_{i l}-\delta_{i k}$ and $S_{i}=S T_{i}$ :

(1) With the quark with charges $S_{i}=\delta_{i l}-\delta_{i j}$ and $\tilde{S}_{\tilde{i}}=0$ in the region

$$
0<\operatorname{Re}\left(\frac{m_{l}-m_{j}}{m_{l}-m_{k}}\right)<1
$$

(2) With the quark with charges $S_{i}=\delta_{i l}$ and $\tilde{S}_{\tilde{i}}=-\delta_{\tilde{i} \tilde{j}}$ in the region

$$
0<\operatorname{Re}\left(\frac{m_{l}-\tilde{m}_{\tilde{j}}}{m_{l}-m_{k}}\right)<1
$$


Notice that the bound-states have exactly the same quantum numbers as the soliton fermion bound-states in Theory A and moreover regions (4.4) and (4.5) are precisely the same as regions (2.36) and (2.37), respectively. At the boundary the bound-state will decay to a dyon and a quark in exactly the same way as in Theory A. We conclude from this that the weak coupling spectrum of topologically charged BPS states is identical in Theory A and B.

While we have shown classical agreement between the masses of the BPS massive hypermultiplet spectrum of Theory B and the the BPS spectrum of Theory A, the spectrum and low-energy interactions of the four-dimensional $\mathcal{N}=2$ supersymmetric gauge theory are corrected by quantum effects. In the asymptotically free case $N_{f}<2 N$ the coupling constant runs at one loop and the complex classical parameter $\tau$ is replaced by the RG invariant scale $\Lambda$. The theory also has an anomaly in the $U(1)$ part of the R-symmetry group which is broken to $Z_{4 N-2 N_{f}}$. This residual discrete symmetry is explicitly broken down to $Z_{2}$ for non-zero hypermultiplet masses. At the baryonic root, Theory B is described by the degenerate elliptic curve,

$$
F(t, v)=\left(t \Lambda^{N-N_{f}} \prod_{\tilde{i}=1}^{N_{f}-N}\left(v-\tilde{m}_{\tilde{i}}\right)-\prod_{i=1}^{N}\left(v-m_{i}\right)\right)\left(t-\Lambda^{N}\right)
$$

This is equivalent to the curve describing the baryonic root given in [4]. All parameters and variables in the curve have even charge under $Z_{4 N-2 N_{f}}$ and thus, as in [4], the effective symmetry of the curve is the $Z_{2}$ quotient $Z_{2 N-N_{f}}=Z_{N-\tilde{N}}$. This form of the curve occurs naturally in the M-theory construction of [3] which we review in Section 5. The curve is branched over the $N$ points $e_{i}$, with $i=1,2, \ldots, N$, defined by,

$$
\prod_{i=1}^{N}\left(v+m_{i}\right)-\Lambda^{N-\tilde{N}} \prod_{\tilde{i}=1}^{\tilde{N}}\left(v+\tilde{m}_{i}\right)=\prod_{i=1}^{N}\left(v-e_{i}\right)=0
$$

Note that this is the same as equation (3.9) which determines the critical points of the twisted superpotential of Theory A.

As in Theory A, the case of vanishing masses $m_{i}=\tilde{m}_{\tilde{i}}=0$ will be of particular interest. In this case, the low-energy theory at the baryonic root has an unbroken $S U(\tilde{N}) \times U(1)^{N-\tilde{N}}$ gauge symmetry [4] with $N_{f}$ massless flavours in the fundamental representation of $S U(\tilde{N})$. Thus the spectrum of the theory includes the corresponding massless quarks and gluons. This is consistent because the condition $N_{f}=N+\tilde{N}<2 N$ which guarantees that the microscopic theory is asymptotically free, implies that $N_{f}>2 \tilde{N}$, which ensures that the low-energy theory is IR free. The massless spectrum at this point also includes $N-\tilde{N}$ hypermultiplets which are neutral under the non-abelian factor of the unbroken gauge group. The theory with zero 
masses also has an unbroken $S U(N-\tilde{N})$ global symmetry and a $Z_{2 N-2 \tilde{N}}$ R-symmetry which is unbroken at the baryonic root.

In the quantum theory the central charge is given by the integral of the Seiberg-Witten differential $\lambda_{S W}=v d(\log t)$ over certain one cycles of the curve. The resulting modification of the classical formula $(4.3)$ is

$$
Z=\sum_{i=1}^{N}\left(m_{i} S_{i}+m_{D i} T_{i}\right)+\sum_{\tilde{i}=1}^{\tilde{N}} \tilde{m}_{\tilde{i}} \tilde{S}_{\tilde{i}}
$$

with

$$
m_{D l}-m_{D k}=\frac{1}{2 \pi i} \int_{e_{k}}^{e_{l}} d \lambda_{\mathrm{SW}}=\frac{1}{2 \pi i} \int_{e_{k}}^{e_{l}} v \frac{d t}{t}
$$

which gives

$$
\begin{aligned}
m_{D l}-m_{D k} & =\frac{1}{2 \pi}\left[\sum_{i=1}^{N} \int_{e_{k}}^{e_{l}} \frac{v d v}{v+m_{i}}-\sum_{\tilde{i}=1}^{N} \int_{e_{k}}^{e_{l}} \frac{v d v}{v+\tilde{m}_{\tilde{i}}}\right] \\
& =\frac{1}{2 \pi}\left[(N-\tilde{N})\left(e_{l}-e_{k}\right)-\sum_{i=1}^{N} m_{i} \log \left(\frac{e_{l}+m_{i}}{e_{k}+m_{i}}\right)+\sum_{\tilde{i}=1}^{N} \tilde{m}_{\tilde{i}} \log \left(\frac{e_{l}+\tilde{m}_{\tilde{i}}}{e_{k}+\tilde{m}_{\tilde{i}}}\right)\right]
\end{aligned}
$$

Thus we have shown that the central charge of Theory B agrees with the exact central charge (3.11,3.12) of Theory A in the previous section.

Although we have complete agreement between the central charges of Theory A and Theory B, it still remains to compare which values of the quantum numbers $S_{i}, \tilde{S}_{\tilde{i}}$ and $T_{i}$ actually appear in the BPS spectra of the two theories. As long as we choose the masses $m_{i}$ so that a sufficient number of gauge bosons have masses much larger than the dynamical scale $\Lambda$, Theory $\mathrm{B}$ is weakly coupled and its spectrum is essentially the classical spectrum described above. Hence, in this regime, massive hypermultiplets of Theory B are in one-to-one correspondence with BPS states of Theory A. However, there is more: the curves of marginal stability at weak coupling in Theory A, where topologically charged BPS solitons decay, have a precise correspondence in Theory B, where the associated magnetically charged dyon states decay. In the gauge theory, these curves correspond to regions were the dimension of the monopole moduli space enlarges discontinuously corresponding to the freedom for the dyon to separate into its decay products [23, 24]. Away from weak coupling, the determination of which states appear in the BPS spectra of either case is complicated by the presence of additional curves of marginal stability. 
Because the exact central charges agree, the same curves of marginal stability occur in both theories. This suggests, but does not guarantee, that the two spectra agree throughout the parameter space. In the next Section we will consider a brane configuration which provides further evidence for this proposal.

One interesting consequence of the proposed equivalence is obtained by setting all twisted masses to zero. This leads to a novel connection between four-dimensional $\mathcal{N}=2$ SQCD with $N$ colours and $N_{f}=N+\tilde{N}$ massless flavours at the baryonic root and the supersymmetric $C P^{N-\tilde{N}}$ $\sigma$-model in two-dimensions. Both theories are asymptotically free and have a $U(1)_{R}$ symmetry which broken to a $Z_{2 N-2 \tilde{N}}$ subgroup by instantons. According to [4] the global symmetry on the baryonic branch is $S U(N-\tilde{N})$ which is the same as the global symmetry of the $C P^{N-\tilde{N}}$ $\sigma$-model. In addition to the massless spectrum of the four-dimensional theory described above, we should find massive hypermultiplet corresponding to each state in the $\sigma$-model spectrum. Thus we predict massive hypermultiplets transforming in the fundamental representation of the global symmetry group as well as bound states transforming in each antisymmetric tensor representation. In Appendix B, we show that anti-symmetric tensor multiplets of the larger symmetry group $S U\left(N_{f}\right)$ exist at weak coupling for zero masses but large VEVs, i.e. not at the baryonic root.

\section{The Brane Configuration}

The technique of realising field theories as the world-volume dynamics of intersecting brane configurations in type II string theory [27] has proved very powerful in recent years, providing a geometrical description of many strong-coupling phenomena in field theory. In fact it is well known how to construct either Theory A or Theory B introduced above on the world-volume of type IIA branes which are subsequently elevated to M-theory. The four-dimensional theory was first studied in this context by Witten in [3], where it was shown that the M5-brane provides a concrete realisation of the Seiberg-Witten curves. The brane model of the two dimensional theory was studied by Hanany and Hori [1]. In the following sections we review both of these constructions and in, particular, how BPS spectrum is realized in each case. In both theories, BPS states correspond to M2 branes with boundaries on M5 branes. In both cases, the relevant boundaries are non-trivial one-cycles on the same Riemann surface.

We look first at the brane picture for the two-dimensional theory described in [1]. The configuration involves a pair of non-parallel NS 5-branes. The first of the pair spans worldvolume directions 012345 and is positioned at $x^{6}=x^{7}=x^{8}=x^{9}=0$. The second NS 5-brane 


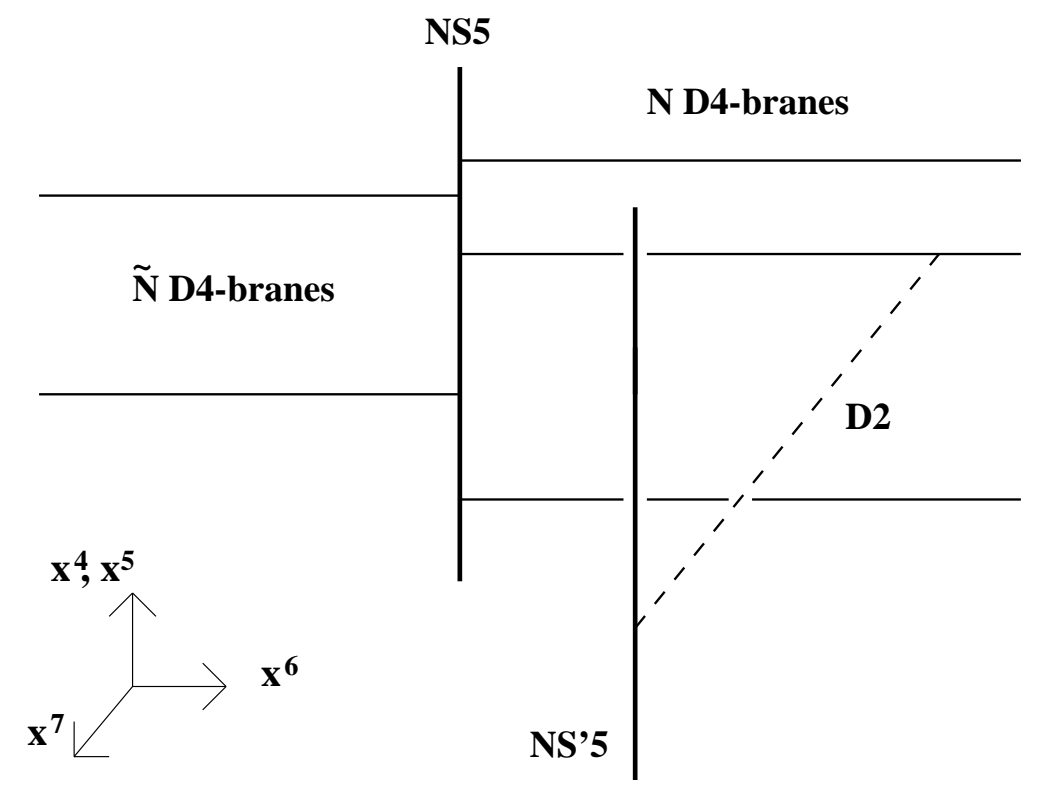

Figure 1: The IIA configuration for theory A

is rotated with respect to the first, spanning world-volume directions 014589. Such a rotation of the second NS 5-brane is typical when constructing theories with four supercharges and this brane is often referred to as an $\mathrm{NS}^{\prime} 5$-brane. It is located at $x^{2}=x^{3}=0$, and at some fixed value of $x^{6}$ and $x^{7}$. The configuration also involves $N+\tilde{N}$ semi-infinite D4-branes ending on the NS 5-brane. Each spans world-volume directions 01236 and is located at $x^{7}=x^{8}=x^{9}=0$ and at a fixed value of $x^{4}$ and $x^{5}$. The D4-branes may end on the NS 5-brane either from the "left" (world-volume $\left.x^{6}<0\right)$ or from the "right" $\left(x^{6}>0\right)$. For our purposes we require $N$ to end from the right, and the remaining $\tilde{N}$ to end from the left. We will refer to the former as D4-branes and the latter as D4-branes. A recent paper which discusses similar configurations of semi-infinite D4-branes is [28].

The $U(1)$ two-dimensional gauge theory is realised as the low-energy dynamics of a single D2-brane suspended between the NS and NS ${ }^{\prime}$ 5-brane, with world-volume coordinates 017 . The D2-brane has finite extent in the $x^{7}$ direction and is infinite in $x^{0}$ and $x^{1}$, these latter dimensions playing the role of the field theory space-time. The final configuration is depicted in figure 1. It preserves four supercharges as required for $\mathcal{N}=(2,2)$ supersymmetry in two-dimensions. Hanany and Hori argue that strings stretched between the D2-brane and semi-infinite D4-branes become chiral multiplets in two dimensions leading to Theory A described in the introduction.

Parameters of the field theory are encoded as positions of the various branes. The VEV of the vector multiplet scalar and the twisted mass parameters both correspond to the positions 
of Dirichlet branes in the $\left(x^{4}, x^{5}\right)$ plane,

$$
\begin{array}{rlrl}
x^{4}+\left.i x^{5}\right|_{\mathrm{D} 2} & =\sigma & \\
x^{4}+\left.i x^{5}\right|_{\mathrm{D} 4_{i}} & =m_{i} & & i=1, \ldots, N \\
x^{4}+\left.i x^{5}\right|_{\tilde{D} 4_{\tilde{i}}} & =\tilde{m}_{\tilde{i}} & & \tilde{i}=1, \ldots, \tilde{N} .
\end{array}
$$

Rotational symmetry in the 45-plane is identified with the $U(1)_{A}$ R-symmetry of the $\mathcal{N}=(2,2)$ theory. The distances between the two 5 -branes in the $x^{6}$ and $x^{7}$ directions are related to the bare FI parameter and bare gauge coupling respectively,

$$
\begin{aligned}
& \frac{\Delta x^{6}}{g_{s t}}=r \\
& \frac{\Delta x^{7}}{g_{s t}}=\frac{1}{e^{2}}
\end{aligned}
$$

where $g_{s t}$ is the string coupling constant, $\Delta x=x\left(\mathrm{NS}^{\prime}\right)-x(\mathrm{NS})$, and we are working in units where the string length-scale $\sqrt{\alpha^{\prime}}$ is set to one. For $\Delta x^{6}=0$, both ends of the D2-brane lie on a 5 -brane, and it is free roam the $\left(x^{4}, x^{5}\right)$ plane. This corresponds to the "Coulomb branch" of the gauge theory. For $\Delta x^{6}>0$, the D2-brane must end on one of the D4-branes and is therefore restricted to lie at one of $N$ fixed positions in $x^{4}$ and $x^{5}$ (this situation is shown in figure 1). For generic mass terms, these positions correspond to the $N$ classical vacua of the field theory for $r>0$ described in Section 2. Similarly, for $\Delta x^{6}<0$ there $\tilde{N}$ choices of D̃ 4 -brane on which the D2-brane may terminate.

While the type IIA picture captures many of the classical properties of the field theory, including the classical moduli space of vacua, quantum effects are reproduced only after raising the configuration to M-theory [1]. As noted in that reference, the field theory limit of M-theory differs from the supergravity limit. Nevertheless we expect certain quantities, in particular masses of BPS states, to be correctly determined in the supergravity approximation. In eleven dimensions the system comprised of the NS 5-brane, D4-branes and $\tilde{D} 4$-branes combines to become a single M5-brane, while the NS' 5-brane evolves into a flat M5-brane and the D2-brane becomes an M2-brane suspended between the two. We deal first with NS 5-brane and D4 (D̃4)branes. The world-volumes of all these branes share the 0123 coordinates and the resulting M5brane is correspondingly flat in these directions. The remaining world-volume directions of the M5-brane describe a two-dimensional curve, $\Sigma$, lying in the submanifold $R^{3} \times S^{1}$, parametrised by $x^{4}, x^{5}, x^{6}$ and periodic coordinate $x^{10}=x^{10}+2 \pi R$. The preservation of eight supercharges requires this curve to be embedded holomorphically with respect to the complex coordinates

$$
v=x^{4}+i x^{5} \quad, \quad s=R^{-3 / 2} x^{6}+i R^{-1} x^{10}
$$


In terms of $t=\Lambda^{N} \exp (-s)$, the curve $\Sigma$ takes the form [1]

$$
t \Lambda^{N-N_{f}} \prod_{\tilde{i}=1}^{\tilde{N}}\left(v-\tilde{m}_{\tilde{i}}\right)=\prod_{i=1}^{N}\left(v-m_{i}\right)
$$

where the product terms can be understood as arising from the deformation of the IIA NS 5-brane due to presence of the D4- and $\tilde{D} 4$-branes.

The description of the NS' 5-brane in M-theory is much simpler. It is not deformed by D4-branes and evolves to a flat M5-brane with world-volume directions 014589. It's position in the $x^{6}$ and $x^{10}$ directions is given by

$$
t=\Lambda^{N}
$$

Unlike the type IIA picture, the M5-branes no longer have a definite $x^{6}$ separation and the quantity $\Delta x^{6}(v)$ is interpreted as the running FI parameter in agreement with field theory [1]. In addition the curved M5 brane configuration is no longer invariant under rotations in the 45-plane. This corresponds to the $U(1)_{A}$ anomaly which appears at one-loop in the twodimensional quantum theory.

The M-theory description of the D2-brane is as an M2-brane, stretched between the flat and curved M5-branes. Like its D2 descendant, the M2-brane world-volume is infinite in the 01 directions and finite in $x^{7}$. Unlike the M5-branes however, the M2-brane does not have a fixed configuration. Rather, different configurations correspond to different states of the twodimensional gauge theory. For example, supersymmetry is unbroken in the vacuum state of the gauge theory and this is reflected in the corresponding M2-brane ground configuration by the requirement that it preserve four supercharges. The only such M2-brane configurations are straight strips lying at a fixed value of $v$ and $t$. It must intersect the two M5-branes and therefore has to lie at $t=\Lambda^{N}$. The possible positions in the $v$-plane are then determined by

$$
\prod_{i=1}^{N}\left(v-m_{i}\right)-\Lambda^{N-\tilde{N}} \prod_{\tilde{i}=1}^{\tilde{N}}\left(v-\tilde{m}_{\tilde{i}}\right)=0
$$

in agreement with field theoretic expectations (3.9). Excited states in the field theory correspond to excited states of the M2-brane, no longer a straight strip but a topologically non-trivial surface with boundaries on the two M5-branes. We will return to these states after first reviewing the four-dimensional theory.

The type IIA realisation of pure $S U(N) \mathcal{N}=2$ SYM consists of $N$ D4-branes suspended between two parallel NS 5-branes. The latter span world-volume directions 012345. Each is 
located at $x^{7}=x^{8}=x^{9}=0$ and at a fixed $x^{6}$ value. The separation of the two NS5-branes in the $x^{6}$ direction determines the bare four-dimensional coupling constant,

$$
\frac{\Delta x^{6}}{g_{s t}}=\frac{1}{g^{2}}
$$

The four-dimensional theory itself lives on the world-volume of the D4-branes, spanning directions 01236. The D4-branes are finite in the $x^{6}$ direction and at large distances their low-energy dynamics are described by $D=4, S U(N) \mathcal{N}=2 \mathrm{SYM}$ with $x^{0}, \ldots, x^{3}$ playing the role of spacetime [3]. The D4-branes are free to move in the $v$-plane and their positions in these directions parametrise the classical Coulomb branch of the theory.

Hypermultiplets transforming in the fundamental representation of the gauge group may be incorporated in one of two ways: using either D6-branes or D4-branes. We choose the latter. The world-volumes of these D4-branes also span directions 01236. Each is attached to one of the two NS 5-branes and are thus semi-infinite in the $x^{6}$ direction. In order to exhibit the similarity to theory A in the most transparent manner, we choose to attach $N$ semi-infinite D4branes to the "right-hand" NS 5-brane. These have world-volume $x^{6} \rightarrow+\infty$. The remaining $\tilde{N}$ D4-branes are attached to the "left-hand" NS 5-brane, have world-volume $x^{6} \rightarrow-\infty$ and, in analogy with theory $\mathrm{A}$, will be referred to as $\tilde{\mathrm{D}} 4$-branes. The position of each semi-infinite D4-brane in the $v$-plane determines the mass of the corresponding hypermultiplet.

$$
\begin{array}{rlrl}
x^{4}+\left.i x^{5}\right|_{\mathrm{D} 4_{i}}=m_{i} & i=1, \ldots, N . \\
x^{4}+\left.i x^{5}\right|_{\tilde{\mathrm{D}} 4_{\tilde{i}}}=\tilde{m}_{\tilde{i}} & i=1, \ldots, N_{f}-N .
\end{array}
$$

As explained in the previous section, the root of the baryonic Higgs branch occurs classically when the VEVs are equal to the masses of some set of $N$ hypermultiplets. The particular brane configuration described above above naturally selects $N$ hypermultiplets as those ending on the "right-hand" NS 5-brane. The root of this baryonic Higgs branch then corresponds to the situation depicted in figure 2 .

As in the two-dimensional case, many quantum aspects of the field theory are uncovered by elevating this configuration to eleven dimensions. Generically, such a system of NS 5-branes and D4-branes becomes a single smooth object in M-theory. The world-volume of this single M5brane spans directions 0123 and describes a two-dimensional surface embedded in $x^{4}, x^{5}, x^{6}$ and $x^{10}$. Once again, the preservation of eight supercharges requires the curve to be holomorphic with respect to the complex structures (5.2).

For the IIA configuration corresponding to an arbitrary point on the Coulomb branch, the lift to M-theory is described by

$$
F(t, v)=A(v) t^{2}+B(v) t+C(v)=0
$$




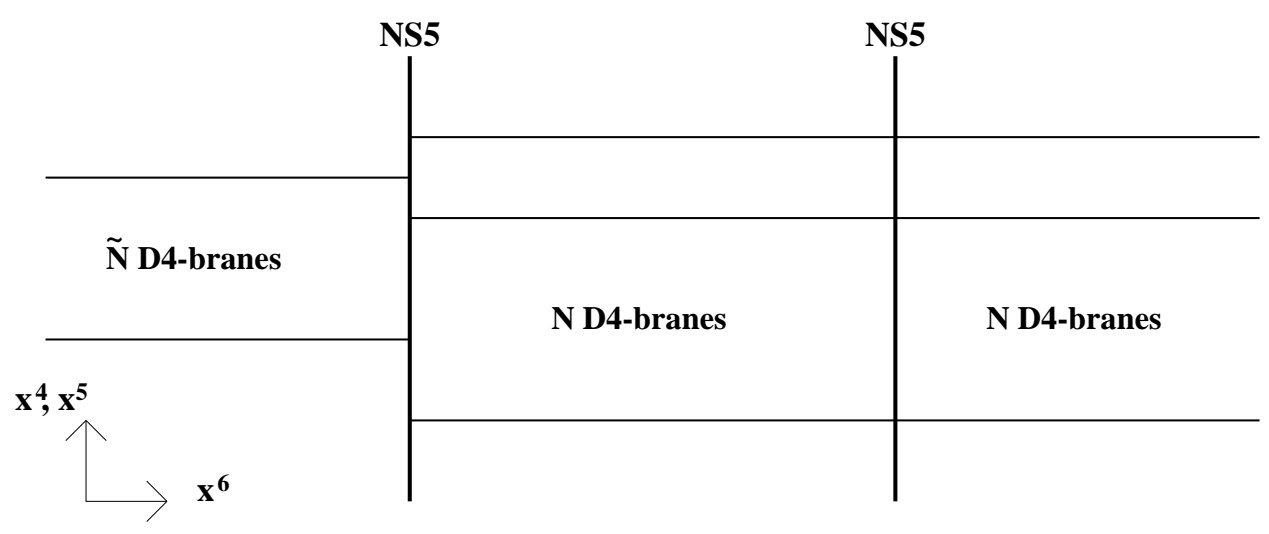

Figure 2: The IIA configuration for theory B

where

$$
\begin{aligned}
& A(v)=\Lambda^{N-N_{f}} \prod_{\tilde{i}=1}^{N_{f}-N}\left(v-\tilde{m}_{\tilde{i}}\right) \\
& B(v)=\prod_{a=1}^{N}\left(v-\phi_{a}\right) \\
& C(v)=\Lambda^{N} \prod_{i=1}^{N}\left(v-m_{i}\right)
\end{aligned}
$$

We will consider the asymptotically free case, $N_{f}<2 N$. The curve branches at $B^{2}-4 A C=0$, defining the $2 N$ branch points, $e_{a}$ and $\tilde{e}_{a}$,

$$
\prod_{a=1}^{N}\left(v-\phi_{a}\right)^{2}-4 \Lambda^{2 N-N_{f}} \prod_{i=1}^{N}\left(v-m_{i}\right) \prod_{\tilde{i}=1}^{N_{f}-N}\left(v-\tilde{m}_{\tilde{i}}\right) \equiv \prod_{a=1}^{N}\left(v-e_{a}\right)\left(v-\tilde{e}_{a}\right)
$$

The root of the baryonic Higgs branch occurs at the point at which a maximal number of cycles vanish. This occurs when $e_{a}=\tilde{e}_{a}$ for all $a=1, \ldots, N$. The solution is given by

$$
B(v)=-\Lambda^{2 N-N_{f}} \prod_{\tilde{i}=1}^{N_{f}-N}\left(v-\tilde{m}_{\tilde{i}}\right)-\prod_{i=1}^{N}\left(v-m_{i}\right)
$$

and the curve becomes,

$$
F(t, v)=\left(t \Lambda^{N-N_{f}} \prod_{\tilde{i}=1}^{N_{f}-N}\left(v-\tilde{m}_{i}\right)-\prod_{i=1}^{N}\left(v-m_{i}\right)\right)\left(t-\Lambda^{N}\right)
$$

This is the curve used in Section 4 to obtain the exact BPS spectrum of Theory B. Importantly, at the root of the baryonic Higgs branch the curve has a very simple factorisation. The single 
M5-brane can be thought of as degenerating into two M5-branes, each described by one of the factors of (5.9). The first factor corresponds to a non-trivial M5-brane, while the second is simply a flat M5-brane located at $t=\Lambda^{N}$. Notice that the first factor is precisely the curve $\Sigma$ describing the NS 5-brane and D4-branes of the two-dimensional theory (5.3). Of course, this is not surprising considering the similarity of the type IIA configurations for theories A and B.

We now turn to the description of BPS states in the two theories, starting with Theory A [1]. States of the field theory correspond to states of the M2-brane. We have already seen how the ground states of the field theory are reproduced by the M2-brane states (5.5) preserving four supercharges. The M-theory realisation of BPS states may be identified in a similar manner as M2-brane configurations preserving two supercharges. In Appendix $\mathrm{C}$ we show that this condition requires the spatial part of the M2-brane world-volume form to be the pull-back of

$$
\omega=\Omega+\mathrm{d} x^{7} \wedge \mathrm{d} x^{1}
$$

where $\Omega$ is the holomorphic two-form

$$
\Omega=\mathrm{d} s \wedge \mathrm{d} v
$$

The mass of such a BPS state is given by the difference between the mass of the corresponding excited M2-brane and the ground M2-brane. Each is given by the area of the brane multiplied by the brane tension, $T$, and may be regulated by introducing a cut-off on the integration over $x^{1}$. The ground state brane, which we denote as $\mathrm{M} 2_{0}$, is at fixed values of both $v$ and $t$ and has world-volume given by the pull-back of $\mathrm{d} x^{7} \wedge \mathrm{d} x^{1}$. The mass of any BPS state is therefore given by

$$
\begin{aligned}
M & =T\left|\int_{\mathrm{M} 2} \omega-\int_{\mathrm{M} 2_{0}} \omega\right| \\
& =T\left|\int_{\mathrm{M} 2} \Omega+\int_{\mathrm{M} 2-\mathrm{M} 2_{0}} \mathrm{~d} x^{1} \wedge \mathrm{d} x^{7}\right| \\
& =T\left|\int_{\mathrm{M} 2} \Omega\right|
\end{aligned}
$$

This formula for the mass of BPS states was also proposed in [1] using different arguments. The mass is seen to be independent of $\Delta x^{7}$ in agreement with field theory expectations that the masses of BPS states do not depend on the gauge coupling constant, e. To make contact with the BPS mass formula of Section 3 , note that $\Omega$ is exact; $\Omega=\mathrm{d} \lambda_{S W}$, where

$$
\lambda_{S W}=v \frac{\mathrm{d} t}{t}
$$


is recognised, in the four-dimensional context, as the Seiberg-Witten differential introduced in Section 4. Using Stokes' theorem, the mass of the BPS states can be rewritten as

$$
M=T\left|\int_{\partial \mathrm{M} 2} \lambda_{S W}\right|
$$

where $\partial \mathrm{M} 2$ is the boundary of the M2-brane. For $\Delta x^{7}=0$, this boundary consists of two curves, each lying on one of the M5-branes. Denote the curve on the flat M5-brane as $C_{r}$ and that on the curved M5-brane as $C_{l}$. Then,

$$
M=T\left|\int_{C_{l}+C_{r}} \lambda_{S W}\right|=T\left|\int_{C_{l}} \lambda_{S W}\right|
$$

where the second equality follows because $\lambda_{S W}$ vanishes over the flat M5-brane (5.4) positioned at a fixed value of $t$. Note that the masses of the BPS states depending only on the details of the curved M5 brane $\Sigma(5.3)$. The M-theory realisation of BPS states in the four-dimensional theory were discussed in detail in [29, 30]. They also correspond to holomorphically embedded M2-branes with world-volume form given by the pull-back of $\Omega$ and with boundary on the M5-branes. The mass of such a state is given by the integral of the Seiberg-Witten differential over the boundary of the M2-brane, in agreement with (5.13). Moreover, the factorisation of the Seiberg-Witten curve at the baryonic root ensures that, as in Theory A, the masses of BPS states are determined solely by a choice of boundary $C_{l}$ on $\Sigma$, the curved part of the M5-brane (5.9).

Although we have shown both in field theory and from branes that the BPS mass formula for the two theories agrees, we would like conclude that the quantum numbers of BPS states appearing in the two theories are the same throughout parameter space. As mentioned in the previous sections, there is no obvious way to do this using conventional field theory methods. In the brane context, determining which BPS states appear is equivalent to determining which BPS configurations of the M2 brane are allowed. In the present case this amounts to determining which one-cycles on the curve $\Sigma$ can arise as boundaries of a BPS M2 brane. At least for small masses, this problem was solved explicitly for Theory A in [1], yielding agreement with previous results for the $C P^{N-1} \sigma$-model. Here we simply note that the equivalent problem for Theory $\mathrm{B}$ is essentially identical and the same analysis should apply. This provides further evidence for the exact agreement of the BPS spectra of the two theories.

Finally, we should comment on the fact that not all BPS states of Theory B appear in spectrum of Theory A. Specifically, only the massive hypermultiplets of Theory B participate 
in the correspondence. The fact that the massless states of Theory B have no counterparts in Theory A corresponds to a general feature of configurations involving branes of different dimensions which was first discussed in [27]: moduli in the higher-dimensional world-volume theory correspond to parameters in the lower-dimensional world-volume theory. By $\mathcal{N}=2$ SUSY all massless fields in Theory B are associated with scalar moduli. As these moduli become parameters in Theory B, we know that the corresponding massless degrees of freedom must decouple from the two-dimensional spectrum. The question of what happens to the massive vector multiplets of Theory $B$ is more subtle as these states have exactly the same masses and charges as some of the hypermultiplets at the baryonic root. Because of this degeneracy, it seems possible that a massive vector multiplet can be interpreted as a threshold boundstate of a massive hypermultiplet and some massless ones. In principle, the existence of such threshold bound-states may be sensitive to deformations of the theory, such as those considered above, which leave the spectrum of truly stable BPS states invariant. Clearly this aspect of the correspondence requires further investigation.

\section{Acknowledgements}

The authors would like to thank Sumit Das, Sunil Mukhi, Sumati Surya, Brett Taylor, Sachin Vaidya and especially Ami Hanany for useful discussions. We would also like to thank Radu Tatar for providing us with a preliminary version of 28]. ND and TJH acknowledge support from the TMR network grant FMRX-CT96-0012.

\section{Appendix A}

In this appendix we give some details of the soliton solutions of the theory in terms of the $(\mathcal{R}, \Theta)$ variables of equation (2.26). From (2.29) we deduce that a BPS saturated soliton solution satisfies the first order equation,

$$
\frac{\partial \Lambda}{\partial x}=\frac{\Delta \mathcal{W}}{|\Delta \mathcal{W}|} g^{\Lambda \bar{\Lambda}} \frac{\partial \overline{\mathcal{W}}}{\partial \Lambda^{\dagger}}=\exp (i \gamma) \frac{|m|}{r} \mathcal{R}(\mathcal{R}-r)
$$

where, $\gamma=\tan ^{-1}(2 \pi r / \Delta \Theta)$. Taking the real and imaginary parts of this equation we obtain,

$$
\frac{\partial \mathcal{R}}{\partial x}=\frac{1}{2 \pi} \tan \gamma \frac{\partial \Theta}{\partial x}=|m| \sin \gamma\left[\frac{\mathcal{R}(r-\mathcal{R})}{r}\right]
$$


The required solution obeys the boundary conditions, $\Lambda \rightarrow 0$ as $x \rightarrow-\infty$ and $\Lambda \rightarrow \Lambda_{+}=$ ir $+\Delta \Theta / 2 \pi$ as $x \rightarrow+\infty$ and is given by,

$$
\mathcal{R}=\frac{r \exp (|m| \sin \gamma x)}{1+\exp (|m| \sin \gamma x)} \quad \Theta=\Delta \Theta \frac{\exp (|m| \sin \gamma x)}{1+\exp (|m| \sin \gamma x)}
$$

which describes a straight line-segment in the $\Lambda$ plane which joins the points $\Lambda=0$ and $\Lambda=\Lambda_{+}$. The resulting central charge is just $Z=2 \Delta \mathcal{W}=i m \Delta \Lambda=i m(i \Delta \mathcal{R}+\Delta \Theta / 2 \pi)$ and BPS mass formula implies that the mass of the soliton is equal to the length of this line-segment

$$
M=|Z|=|m| \sqrt{r^{2}+\left(\frac{\Delta \Theta}{2 \pi}\right)^{2}}
$$

where we have used $\Delta \mathcal{R}=r$. To interpret the meaning of quantity $\Delta \Theta$ we note that, in the $(\Theta, \alpha)$ variables of (2.18), the Noether charge, $S$, of the global $U(1)$ symmetry of the theory is given as,

$$
S=\frac{r}{2} \int_{-\infty}^{+\infty} d x \sin ^{2} \varphi \dot{\alpha}+\frac{\theta}{4 \pi} \int_{-\infty}^{+\infty} d x \partial_{x}(\cos \varphi)
$$

On the other hand from (A.3) and (2.27) we have,

$$
\frac{1}{2 \pi} \frac{\partial \Theta}{\partial x}=\frac{r}{2} \sin ^{2} \frac{\varphi}{2} \frac{\partial \alpha}{\partial t}
$$

on integrating this equation we find,

$$
\frac{\Delta \Theta}{2 \pi}=\frac{r}{2} \int_{-\infty}^{\infty} d x \sin ^{2} \varphi \frac{\varphi}{2} \frac{\partial \alpha}{\partial t}=S+\frac{\theta}{2 \pi}
$$

Thus we recover the central charge (2.22) and the mass formula (2.21),

$$
M=|m| \sqrt{\left(S+\frac{\theta}{2 \pi}\right)^{2}+r^{2}}
$$

\section{Appendix B}

In this appendix, we construct the weak coupling spectrum of bound-states of dyons and quarks in an $\mathcal{N}=2$ supersymmetric $S U(N)$ gauge theory with $N_{f}$ hypermultiplets. Consider the monopole solution with topological (magnetic) charge $h_{a}=\delta_{a c}-\delta_{a b}$. At a generic point in the space of Higgs VEVs, the moduli space of such solutions will be completely described by embedding an $S U(2)$ monopole solution in the $S U(N)$ gauge group [23]. In our analysis, we shall 
avoid submanifolds of co-dimension one, where the space of solutions enlarges discontinuously. These submanifolds correspond to regions where some $\phi_{d}$ lies on the line segment joining $\phi_{b}$ with $\phi_{c}$ and are surfaces on which dyons can decay to other dyons.

Before we proceed, we find it useful to introduce the roots and weights of $S U(N)$. To this end, we introduce $\boldsymbol{e}_{a}, a=1, \ldots, N$, the weights of the $\boldsymbol{N}$ representation. The roots are then $\boldsymbol{e}_{a}-\boldsymbol{e}_{b}$. The monopole that we are considering has a topological charge equal to the root $\boldsymbol{h} \equiv \boldsymbol{\alpha}=\boldsymbol{e}_{c}-\boldsymbol{e}_{b}$. We now analyse the time-independent Dirac equation for the fermion field $\Psi_{a}^{\lambda}$, in the hypermultiplet $\left\{Q_{a}^{\lambda}, \tilde{Q}_{\lambda}^{a}\right\}$, in the background of the monopole. The resulting equation is only non-trivial if $a=b$ or $a=c$ and without loss of generality we can choose $a=b$. The Dirac equation is given by a generalization of that written down in [23] which includes an explicit mass term:

$$
\left(\begin{array}{cc}
S & -\mathcal{D}^{*} \\
-\mathcal{D} & -S
\end{array}\right) \Psi_{b}^{\lambda}=E \Psi_{b}^{\lambda}
$$

Here

$$
\mathcal{D}=-i \vec{\sigma} \cdot \vec{D}+i P, \quad D^{*}=-i \vec{\sigma} \cdot \vec{D}-i P
$$

where $\vec{\sigma}$ are the Pauli sigma matrices and $\vec{D}$ is the spatial part of the covariant derivative in the background of the monopole solution. The other quantities in (B.1) and (B.2) are

$$
\begin{aligned}
P & =\phi^{c} t^{c}+\operatorname{Re}\left(e^{-i \sigma} \boldsymbol{\phi}\right) \cdot \boldsymbol{H}-\frac{|\boldsymbol{\phi} \cdot \boldsymbol{\alpha}|}{\boldsymbol{\alpha}^{2}} \boldsymbol{\alpha} \cdot \boldsymbol{H}+\operatorname{Re}\left(e^{-i \sigma} m_{\lambda}\right) \\
S & =\operatorname{Im}\left(e^{-i \sigma} \boldsymbol{\phi}\right) \cdot \boldsymbol{H}+\operatorname{Im}\left(e^{-i \sigma} m_{\lambda}\right)
\end{aligned}
$$

where $\sigma$ is the phase of $\boldsymbol{\phi} \cdot \boldsymbol{\alpha}$, the $t^{c}$ are the generators of the $S U(2)$ corresponding to the root $\boldsymbol{\alpha}, \phi^{c}$ is the Higgs field of the $S U(2)$ BPS monopole and $\boldsymbol{H}$ are the Cartan generators of $S U(N)$.

We will now show that BPS bound-states of the monopole, and its dyonic excitations, with the quark exists whenever $\mathcal{D}$ has a normalizable zero-mode. Suppose that such a mode $\psi_{b}^{\lambda}$ exists. This implies that the Dirac operator has an eigenvector:

$$
\left(\begin{array}{cc}
S & -\mathcal{D}^{*} \\
-\mathcal{D} & -S
\end{array}\right)\left(\begin{array}{c}
\psi_{b}^{\lambda} \\
0
\end{array}\right)=\operatorname{Im}\left(e^{-\sigma} \boldsymbol{\phi} \cdot \boldsymbol{e}_{b}+e^{-\sigma} m_{\lambda}\right)\left(\begin{array}{c}
\psi_{b}^{\lambda} \\
0
\end{array}\right)
$$

where we used the fact that $\boldsymbol{H} \Psi_{b}^{\lambda}=\boldsymbol{e}_{b} \Psi_{b}^{\lambda}$. To show that the bound-state is BPS saturated, we notice that although the mode is not a zero-mode of the full Dirac operator, but only of $\mathcal{D}$, it contributes to the central charge in just such a way as to preserve the BPS condition. The central charge of the bound-state is $Z=\tau \boldsymbol{\phi} \cdot \boldsymbol{\alpha}+\boldsymbol{\phi} \cdot \boldsymbol{e}_{a}+m_{\lambda}$ and hence the BPS mass is

$$
M=\left|\tau \boldsymbol{\phi} \cdot \boldsymbol{\alpha}+\boldsymbol{\phi} \cdot \boldsymbol{e}_{b}+m_{\lambda}\right|=|\tau \boldsymbol{\phi} \cdot \boldsymbol{\alpha}|+\operatorname{Im}\left(e^{-i \sigma} \boldsymbol{\phi} \cdot \boldsymbol{e}_{b}+e^{-i \sigma} m_{\lambda}\right)+\cdots
$$


Here the first term is the mass of the monopole, the second term is precisely the energy of the fermion mode and the ellipsis are corrections in $g$ which encode the back-reaction of the fermion fields on the monopole that we have not accounted for in our simple analysis. Notice the this line of reasoning is analogous to our analysis of the soliton-fermion bound-states in Theory A. The semi-classical description of the bound-state is completely standard (see for example [31). The fermion mode is associated to a pair of raising and lowering operators $\rho$ and $\rho^{\dagger}$ with canonical anti-commutation relation $\left\{\rho, \rho^{\dagger}\right\}=1$. The monopole carries a Fock space representation of these operators, in other words there is a vacuum state $|0\rangle$, which represents the original monopole, and an excited state $\rho^{\dagger}|0\rangle$, which represents the bound-state. In addition, the monopole wavefunction also includes the usual dyonic excitation piece which implies that each bound-state gives rise to a tower of states with magnetic charge $\boldsymbol{h}=\alpha$, electric charge $\boldsymbol{q}=S \boldsymbol{\alpha}+\boldsymbol{e}_{b}$, for an integer $S$, and global charge $s_{\mu}=\delta_{\mu \lambda}$.

So our problem is now reduced to searching for normalizable zero-modes of $\mathcal{D}$. Fortunately this analysis is a simple generalization of that in [23]. There exists a single normalizable zeromode if

$$
-1<\operatorname{Re}\left(\frac{\boldsymbol{\phi} \cdot\left(\boldsymbol{e}_{b}+\boldsymbol{e}_{c}\right)+2 m_{\lambda}}{\boldsymbol{\phi} \cdot \boldsymbol{\alpha}}\right)<1
$$

We now analyse this condition at the baryonic root, where $\phi_{a} \equiv \boldsymbol{\phi} \cdot \boldsymbol{e}_{a}=-m_{i} \delta_{i a}$. Furthermore, in order to facilitate comparison with Theory A, we shall write the topological charge of the dyon as $\boldsymbol{\alpha}=\boldsymbol{e}_{k}-\boldsymbol{e}_{l}$, and so $\boldsymbol{\phi} \cdot \boldsymbol{\alpha}=m_{l}-m_{k}$, and the fermion to have mass $m_{\lambda}$ equal to (i) $m_{j}$ (ii) $\tilde{m}_{\tilde{j}}$. For the first case the region where the bound-state exists is

$$
0<\operatorname{Re}\left(\frac{m_{l}-m_{j}}{m_{l}-m_{k}}\right)<1
$$

with quantum numbers $T_{i}=\delta_{i l}-\delta_{i k}, S_{i}=S T_{i}+\delta_{i l}-\delta_{i j}$ and $\tilde{S}_{\tilde{i}}=0$. While in the second case the bound-state exists in a region

$$
0<\operatorname{Re}\left(\frac{m_{l}-\tilde{m}_{\tilde{j}}}{m_{l}-m_{k}}\right)<1
$$

with quantum numbers $T_{i}=\delta_{i l}-\delta_{i k}, S_{i}=S T_{i}+\delta_{i l}$ and $\tilde{S}_{\tilde{i}}=-\delta_{\tilde{i} \tilde{j}}$. In overlapping regions (B.7) and $(\mathbb{B} .8)$ there will be multiple bound-states obtaining by filling out the states of a higher dimensional Fock space.

It is also interesting to analysis the spectrum of bound-state with zero masses $m_{\lambda}=0$. In this case the condition for the existence of a bound-state is

$$
0<\operatorname{Re}\left(\frac{\phi_{l}}{\phi_{l}-\phi_{k}}\right)<1
$$


In this region, there are $N_{f}$ degenerate fermion modes $\psi_{l}^{\lambda}, \lambda=1, \ldots, N_{f}$, and the bound-states are composed of a Fock space representation of the $N_{f}$ creation and annihilation operator $\rho_{\lambda}$ and $\rho_{\lambda}^{\dagger}$. The states are therefore of the form

$$
\rho_{\lambda_{1}}^{\dagger} \cdots \rho_{\lambda_{p}}^{\dagger}|0\rangle \quad \lambda_{i} \neq \lambda_{j}
$$

For a given $p$, these states transform in the $p^{\text {th }}$ anti-symmetric representation of the unbroken $S U\left(N_{f}\right)$ flavour symmetry group.

\section{Appendix C}

In this appendix, using the methods of [32], we derive the world-volume form (5.10) for a M2brane preserving two supercharges. We follow closely the analogous calculation for BPS states in four-dimensional $\mathcal{N}=2$ theories performed by Fayyazuddin and Spaliński [33]. A convenient choice of eleven dimensional gamma matrices $\Gamma_{M}$ satisfying $\left\{\Gamma_{M}, \Gamma_{N}\right\}=2 \delta_{M N}$ is given in the appendix of this reference, such that $\Gamma_{M}$ are real for $M=2,5,7,8,10$ and purely imaginary for other space-time indices and all gamma matrices with spatial indices are hermitian.

The number of supersymmetries preserved by an p-brane with embedding $X^{M}$ in $R^{1,9} \times S^{1}$ is equal to the number of solutions to

$$
\eta=\frac{1}{p !} \epsilon^{\alpha_{1} \ldots \alpha_{p}} \Gamma_{M_{1} \ldots M_{p}} \partial_{\alpha_{1}} X^{M_{1}} \ldots \partial_{\alpha_{p}} X^{M_{p}}
$$

where $\Gamma_{M_{1} \ldots M_{p}}=\Gamma_{\left[M_{1} \ldots \Gamma_{\left.M_{p}\right]}\right.}$ and $\eta$ is an eleven dimensional Majorana spinor (64 real components) which can be decomposed as

$$
\eta=\chi+B \chi^{\star}
$$

where $B$ is equal to the product of the real gamma matrices and determines the charge conjugation matrix $C=B \Gamma_{0}$. We first consider solutions to (C.1) in the background of the M5-branes. The NS 5-brane and D4-brane system lifts to the single M5-brane described by the curve (5.3). It was shown explicitly in [33] that this preserves eight supercharges satisfying

$$
\begin{aligned}
\Gamma_{\bar{v}} \chi=\Gamma_{\bar{s}} \chi & =0 \\
i \Gamma_{0} \Gamma_{1} \Gamma_{2} \Gamma_{3} \chi & =-\chi
\end{aligned}
$$

where $\Gamma_{\bar{v}}$ and $\Gamma_{\bar{s}}$ are the gamma matrices in the complex basis (5.2). The flat M5-brane enforces a further condition on the spinor: $\Gamma_{0} \Gamma_{1} \Gamma_{4} \Gamma_{5} \Gamma_{8} \Gamma_{9} \eta=\eta$. Using the first equation in (C.2), it is simple to show that this is equivalent to

$$
i \Gamma_{0} \Gamma_{1} \Gamma_{8} \Gamma_{9} \chi=-\chi
$$


There are four real independent solutions to (C.2) and (C.3), equivalent to $\mathcal{N}=(2,2)$ supersymmetry in two dimensions. Further, using the fact that the product of all gamma matrices is proportional to the identity matrix, any spinor $\chi$ satisfying both these conditions must also satisfy

$$
\Gamma_{0} \Gamma_{1} \Gamma_{7} \chi=\chi
$$

This reflects the fact that we may add a flat M2-brane to the configuration while preserving four supercharges. This M2-brane corresponds to the ground state of the two-dimensional field theory.

Each state in the field theory other than the vacuum corresponds to an excited M2-brane which is no longer embedded at a fixed value of $v$ and $s$. The condition that this state be BPS requires the existence of a simultaneous solution to (C.2), (C.3) and

$$
\eta=\frac{1}{2} \epsilon_{\alpha \beta} \Gamma_{0} \Gamma_{M N} \partial_{\alpha} X^{M} \partial_{\beta} X^{N} \eta
$$

Introducing the complex coordinate $u=x^{1}+i x^{7}$, this condition becomes

$$
\begin{aligned}
\epsilon_{\alpha \beta} \eta= & \Gamma_{0}\left\{\left(\partial_{\alpha} s \partial_{\beta} v-\partial_{\alpha} v \partial_{\beta} s\right) \Gamma_{s v}+\left(\partial_{\alpha} \bar{s} \partial_{\beta} \bar{v}-\partial_{\alpha} \bar{v} \partial_{\beta} \bar{s}\right) \Gamma_{\bar{s} \bar{v}}\right. \\
& +\left(\partial_{\alpha} s \partial_{\beta} u-\partial_{\alpha} u \partial_{\beta} s\right) \Gamma_{s u}+\left(\partial_{\alpha} \bar{s} \partial_{\beta} \bar{u}-\partial_{\alpha} \bar{u} \partial_{\beta} \bar{s}\right) \Gamma_{\bar{s} \bar{u}} \\
& +\left(\partial_{\alpha} v \partial_{\beta} u-\partial_{\alpha} u \partial_{\beta} v\right) \Gamma_{s v}+\left(\partial_{\alpha} \bar{v} \partial_{\beta} \bar{u}-\partial_{\alpha} \bar{u} \partial_{\beta} \bar{v}\right) \Gamma_{\bar{v} \bar{u}} \\
& +\left(\partial_{\alpha} s \partial_{\beta} \bar{u}-\partial_{\alpha} \bar{u} \partial_{\beta} s\right) \Gamma_{s \bar{u}}+\left(\partial_{\alpha} \bar{s} \partial_{\beta} u-\partial_{\alpha} u \partial_{\beta} \bar{s}\right) \Gamma_{\bar{s} u} \\
& +\left(\partial_{\alpha} v \partial_{\beta} \bar{u}-\partial_{\alpha} \bar{u} \partial_{\beta} v\right) \Gamma_{v \bar{u}}+\left(\partial_{\alpha} \bar{v} \partial_{\beta} u-\partial_{\alpha} u \partial_{\beta} \bar{v}\right) \Gamma_{\bar{v} u} \\
& +i\left(\partial_{\alpha} s \partial_{\beta} \bar{s}+\partial_{\alpha} v \partial_{\beta} \bar{v}-\partial_{\alpha} \bar{s} \partial_{\beta} s-\partial_{\alpha} \bar{v} \partial_{\beta} v\right) \Gamma_{0} \Gamma_{1} \Gamma_{2} \Gamma_{3} \\
& \left.+\left(\partial_{\alpha} u \partial_{\beta} \bar{u}-\partial_{\alpha} \bar{u} \partial_{\beta} u\right) \Gamma_{u \bar{u}}\right\} \eta
\end{aligned}
$$

Mercifully many of these terms vanish upon applying the projection operator $Q=\Gamma_{s} \Gamma_{\bar{s}} \Gamma_{v} \Gamma_{\bar{v}}$, and we are left with

$$
\begin{aligned}
\epsilon_{\alpha \beta} B \chi^{\star}= & \left(\partial_{\alpha} s \partial_{\beta} v-\partial_{\alpha} v \partial_{\beta} s\right) \Gamma_{0} \Gamma_{s} \Gamma_{v} \chi-i\left(\partial_{\alpha} u \partial_{\beta} \bar{u}-\partial_{\alpha} \bar{u} \partial_{\beta} u\right) B \chi^{\star} \\
& +i\left(\partial_{\alpha} s \partial_{\beta} \bar{s}+\partial_{\alpha} v \partial_{\beta} \bar{v}-\partial_{\alpha} \bar{s} \partial_{\beta} s-\partial_{\alpha} \bar{v} \partial_{\beta} v\right) \Gamma_{0} B \chi^{\star}
\end{aligned}
$$

Without the second term on the right-hand side, this is the equation determining the M2-brane realisation of a BPS state in the four-dimensional $\mathcal{N}=2$ theory. In the two-dimensional model under consideration, the presence of this second term reflects the spatial extension of the M2brane in the $x^{1}$ and $x^{7}$ directions. Notice that any term appearing in (C.4) that contained both $u$ and either $s$ or $v$ is eliminated by the projection. Finally, we act with a further projection, $P=\frac{1}{2}\left(1+i \Gamma_{0} \Gamma_{1} \Gamma_{2} \Gamma_{3}\right)$, to arrive at the requirement

$$
B \chi^{\star}=\Gamma_{0} \Gamma_{s} \Gamma_{v} \chi
$$


which is identical to the four-dimensional BPS condition [33]. Moreover, the world-volume form on the BPS M2-brane is determined to be

$$
\epsilon_{\alpha \beta}=\partial_{\alpha} s \partial_{\beta} v-\partial_{\alpha} v \partial_{\beta} s-i \partial_{\alpha} u \partial_{\beta} \bar{u}+i \partial_{\alpha} \bar{u} \partial_{\beta} u
$$

which is indeed the pull-back of the two form $\omega$ given in equation (5.10).

\section{References}

[1] A. Hanany and K. Hori, "Branes and N=2 Theories in Two Dimensions" hep-th/9707192 Nucl. Phys. B513 (1998) 119.

[2] N. Dorey. "The BPS Spectra of Two-Dimensional Supersymmetric Gauge Theories with Twisted Mass Terms", hep-th/9806056, JHEP 11 (1998) 005.

[3] E. Witten, "Solutions Of Four-dimensional Field Theories Via M Theory," hep-th/9703166, Nucl.Phys. B500 (1997) 3.

[4] P. Argyres, M. R. Plesser and N. Seiberg "The Moduli Space of Vacua of N=2 SUSY QCD and Duality in N=1 SUSY QCD", hep-th/9603142, Nucl. Phys. B471 (1996) 159.

[5] S. Cecotti, P. Fendley, K. Intriligator, and C. Vafa, "A New Supersymmetric Index", hep-th/9204102, Nucl.Phys. B386 (1992) 405.

[6] S. Cecotti and C. Vafa, "On Classification of N=2 Supersymmetric Theories", hepth/9211097, Commun. Math. Phys. 158 (1993) 569.

[7] A. Bilal and F. Ferrari, "The Strong-Coupling Spectrum of the Seiberg-Witten Theory", hep-th/9602082, Nucl.Phys. B469 (1996) 387, and "Curves of Marginal Stability and Weak and Strong-Coupling BPS Spectra in $N=2$ Supersymmetric QCD", hep-th/9605101, Nucl. Phys. B480 (1996) 589, and "The BPS Spectra and Superconformal Points in Massive N=2 Supersymmetric QCD", hep-th/hep-th/9706145 Nucl. Phys. B516 (1998) 175.

[8] E. Abdalla, M. Forger and A. Lima Santos, "Nonlocal Charges for Nonlinear Sigma Models on Grassmannian Manifolds", Nucl. Phys. B256 (1985) 145.

E. Abdalla and A. Lima-Santos, "On Some Features of $C P^{n-1}$ Models with Fermions", Phys. Rev. D 29 (1984) 1851.

R. Köberle and V. Kurak, "Solitons in the Supersymmetric $C P^{N-1}$ Model", Phys. Rev. D 36 (1987) 627. 
[9] J. M. Evans and T. J. Hollowood, "The Exact Mass-Gap of the Supersymmetric $C P^{n-1}$ Sigma Model” ,Phys. Lett. B343 (1995) 198.

[10] E. Witten, "Phases of $\mathrm{N}=2$ Theories in Two Dimensions" hep-th/9301042 Nucl. Phys. B403 (1993) 159.

[11] M. Rocek and E. Verlinde, "Duality, Quotients, and Currents", hep-th/9110053, Nucl. Phys. B373 (1992) 630.

[12] D. R. Morrison and M. R. Plesser, "Summing the Instantons: Quantum Cohomology and Mirror Symmetry in Toric Varieties", hep-th/9412236, Nucl. Phys. B440 (1995) 279 and "Towards Mirror Symmetry as Duality for Two-Dimensional Abelian Gauge Theories" hep-th/9508107, Nucl. Phys. Proc. Suppl 46 (1996) 177.

[13] S. Coleman, "There are no Goldstone Bosons in Two Dimensions", Commun. Math. Phys. 31 (1973) 259.

[14] E. Witten and D. I. Olive, "Supersymmetry Algebras that Include Topological Charges", Phys. Lett. B78 (1978) 97.

[15] E. Witten, "Dyons of Charge e $\theta / 2 \pi "$, Phys. Lett. B86 (1979) 283.

[16] P. Fendley, S. D. Mathur, C. Vafa and N. P. Warner, "Integrable Deformations and Scattering Matrices for the N=2 Supersymmetric Discrete Series", Phys. Lett. B243 (1990) 257.

[17] E. Tomboulis and G. Woo, "Soliton Quantization in Gauge Theories", Nucl. Phys. B107 (1976) 221.

[18] B. Julia and A. Zee, "Poles with both Magnetic and Electric Charges in Nonabelian Gauge Theory", Phys. Rev. D11 (1975) 2227.

[19] S. Coleman, "More about the Massive Schwinger Model", Ann. Phys. 101 (1976) 239.

[20] A. D'Adda, A.C. Davis, P. DiVecchia and P. Salomonson, "An Effective Action for the Supersymmetric $C P^{n-1}$ Model", Nucl. Phys. B222 (1983) 45.

[21] E. Witten, "Instantons, the Quark Model, and the 1/N Expansion" Nucl. Phys. B149 (1979) 285.

[22] M. Cederwall and M. Holm "Monopole and Dyon Spectra in N=2 SYM with Higher Gauge Groups", hep-th/9603134. 
[23] T. Hollowood, "Semi-Classical Decay of Monopoles in N=2 Gauge Theory", hepth/9611106.

[24] T.J. Hollowood, "Dyon Electric Charge and fermion Fractionalization in N=2 Gauge Theory", hep-th/9705041 Nucl. Phys. B517 (1998) 161.

[25] J. P. Gauntlett and J. A. Harvey, "S-Duality and the Dyon Spectrum in N=2 Super YangMills Theory", hep-th/9508156 Nucl. Phys. B463 (1996) 287.

[26] S. Sethi, M. Stern and E. Zaslow, "Monopole and Dyon Bound States in N=2 Supersymmetric Yang-Mills Theories", hep-th/9508117 Nucl. Phys. B457 (1995) 484.

[27] A. Hanany and E. Witten, "Type IIB Superstrings, BPS Monopoles and ThreeDimensional Gauge Dynamics", hep-th/9611230, Nucl. Phys. B492 (1997) 152.

[28] K. Oh and R. Tatar, "M-theory Interpretation of Configurations with Semi-Infinite D4branes", hep-th/9902119.

[29] M. Henningson and P. Yi "Four-dimensional BPS-spectra via M-theory", hep-th/9707251, Phys.Rev. D 57 (1998) 1291.

[30] A. Mikhailov "BPS States and Minimal Surfaces", hep-th/9708068, Nucl.Phys. B533 (1998) 243.

[31] J.A. Harvey, "Magnetic Monopoles, Duality and Supersymmetry" hep-th/9603086, Trieste HEP Cosmology (1995) 66.

[32] K. Becker, M. Becker and A. Strominger, "Fivebranes, Membranes and Non-Perturbative String Theory" hep-th/9509175 Nucl. Phys. B456 (1995) 130.

[33] A. Fayyazuddin and M. Spaliński "The Seiberg-Witten Differential from M-Theory" hepth/9706087, Nucl.Phys. B508 (1997) 219. 